\title{
Thermal variational principle and gauge fields
}

\author{
York Schröder* \\ DESY, Theory Group, Notkestrasse 85, D-22603 Hamburg, Germany
}

Hermann Schulz ${ }^{\dagger}$

Institut für Theoretische Physik, Universität Hannover, Appelstrasse 2, D-30167 Hannover, Germany

(Received 9 April 1996)

\begin{abstract}
A Feynman-Jensen version of the thermal variational principle is applied to hot gauge fields, Abelian as well as non-Abelian: scalar electrodynamics (without scalar self-coupling) and the gluon plasma. The perturbatively known self-energies are shown to derive by variation from a free quadratic ("Gaussian") trial Lagrangian. Independence of the covariant gauge-fixing parameter is reached (within the order $g^{3}$ studied) after a reformulation of the partition function such that it depends on only even powers of the gauge field. Also static properties (Debye screening) are reproduced this way. But because of the present need to expand the variational functional, the method falls short of its potential nonperturbative power. [S0556-2821(96)02424-1]
\end{abstract}

PACS number(s): 11.10.Wx, 11.15.Tk

\section{INTRODUCTION}

Variational principles, well established in nonrelativistic quantum problems, develop their true power by setting a measure for the best approximate solution within some parametrization of a trial space. This space is made up of wave functions $\psi$ in nonrelativistic quantum mechanics, by statistical operators $\rho$ in thermodynamics, and by actions $S$ in the Feynman-Jensen formulation. The above object $\psi$ can always be understood to be the ground state of some Hamiltonian $H$. Any statistical operator is also related uniquely to a Hermitian operator $H$ (its "Hamiltonian"). Thus, in any of these three cases, we may talk about a theory to be parametrized and varied. The task is to find a class of theories coming reasonably close to the truth but to keep it simple enough for tractability.

Each of the above three cases generalizes to field theory, $\psi$ becoming a wave functional, see, e.g. [1], while $H$ and $S$ keep their meaning. For the formulations of the thermal variational principle, with $H$ [2-10] or with $S[5,10-15]$, we refer to the next section as some part II of the Introduction.

We focus on the application to pure gauge theory with particular interest in the hot gluon system. There are three fascinating aspects of this system. First, it distills out from the standard model by reducing the number of flavors to zero, while possibly still containing the whole non-Abelian mystery. Second, other than in the Higgs sector (the other distillate) its Lagrangian looks so simple: $\mathcal{L}=-F^{2} / 4$. Third, its high-temperature limit may be called understood, mainly based on the rearrangement $[16,17]$ of diagrams at soft-scale outer momenta $Q$ (key words: resummation, hard thermal loops).

There are several basic problems and pitfalls at the very beginning when the variational method contacts gauge fields. It is the subject of the present paper to make the calculus working at all. Hence no new results on the hot gluon system

\footnotetext{
*Electronic address: yschroed@x4u2.desy.de

†Electronic address: hschulz@itp.uni-hannover.de
}

should be expected here. Instead, the known perturbative results are used to test our new variational construction. With this first step we hope to pave the road towards its presumedly powerful nonperturbative possibilities.

Specifically on $\mathrm{SU}(N)$ gauge fields under thermal variation, there is (to our knowledge) only the one (thus pioneering) paper of Manka [6] in 1986. He studied pure nonAbelian gauge theory by using a free trial Lagrangian, namely, the Abelian one for photons taken $n$-fold $\left(n \equiv N^{2}-1\right)$. The fields are identified, $A_{\text {non-Abelian }}=A_{\text {trial }}$ (at least in the high-temperature phase), and a constant transverse photon mass is taken as variational parameter. Note that this identification of pure, but interacting gauge fields with free, but massive ones makes the trial theory nontrivial. With the longitudinal mass included as well as the fourvertex (which both were neglected in [6]), Manka conjectures that the perturbative results on masses, generated by the plasma, should be obtained from variation as well. Indeed, they should - but by this supposedly easy task we were led into all that follows.

The following outlook reflects, to some extent, our individual path into the subject. Starting with the basic ideas of [6] just mentioned, we were more or less forced into one step (away from [6]) after the other.

(1) The covariant gauge-fixing parameter $\alpha$ is reintroduced, and kept arbitrary, because all experience with, e.g., the damping puzzle of the gluon plasma tells us that $\alpha$, if surviving in final results, is an ideal indicator for wrong physics.

(2) Both dynamical mass terms (transverse and longitudinal) are included as functions of momentum. This setup covers static screening as well as dynamically generated masses. The massive-photonic trial theory still keeps its Abelian gauge invariance.

(3) The functional integral formulation is applied. In passing, although our notation is Minkowskian (metrics +--- ) we actually always mean the Euclidean space. We only have to remember, at appropriate places, that the zeroth component $A^{0}$ of the gauge field is $i$ times a real field.

(4) The classical (or Feynman-Jensen) version of the 
variational functional is used, because it avoids difficulties in constructing the Hamiltonian to our higher-derivative trial Lagrangian. As an intermediate result, the covariant gaugefixing parameters of studied and trial theory become equal.

(5) The variational functional, if evaluated with the quadratic photonic trial theory as described, still depends on the (common) gauge-fixing parameter $\alpha$ (as also observed in [18]). A way out is proposed by first rewriting the partition function of the theory studied such that its action becomes even in the gauge fields. This is called the "even version" in Sec. II C.

(6) As in the low-order perturbative treatments, and since we shall only reproduce its results, detailed renormalization is not (yet) required in this paper. Divergent terms can be separated from the finite thermal ones. Hence, the coupling $g$ changes its meaning to be the running coupling in these thermal contributions.

(7) For a first application of the "even version," scalar electrodynamics (ED) is appreciated once more [19] to be an ideal toy model for the non-Abelian problem. The known self-energies are put in by hand, but supplied with variable prefactors. Through variation, the latter become 1 indeed.

(8) In the non-Abelian case, the Faddeev-Popov determinant becomes part of the even-odd decomposition. The "even" functional works well, except for a (hopefully) minor detail at the end (concerning gauge-fixing dependence in higher order).

(9) For the explicit analysis just mentioned, the variational functional had to be expanded up to the third (partly fourth) $g$ power. This apparently inevitable recourse to $g$ powers is a big disappointment.

The paper is organized as follows. Section II on the formulations of the thermal variational functional is a continued Introduction. Especially the "even version" (the one that works) is introduced in Sec. II C. In Sec. III we follow the Feynman-Jensen version. It leads to unphysical results, but is, on the other hand, reasonably simple to introduce several technical details. Section IV treats scalar electrodynamics with the "even version" of the functional. In Sec. V on the gluon plasma, things start more involved but become very similar at the end. In Sec. VI the case of constant trial selfenergies is discussed in terms of Debye screening and magnetic mass. Open questions are summarized in Sec. VII. Conclusions follow in Sec. VIII. Three Appendices cover details on the functional integral measures, on some normal integrals involved, and on sum rules.

\section{THE THERMAL VARIATIONAL PRINCIPLE}

\section{A. Gibbs-Bogoljubov}

The extremal properties of thermodynamic potentials are known from textbooks $[2,3]$ on statistical physics. In particular in the canonical ensemble (the only one considered in this paper), the free energy takes its minimum at equilibrium: $F \geqslant F$. In its usual version, the thermal variational principle is identical with this modest inequality, if its left-hand side is detailed:

$$
\mathcal{V}[H] \equiv \operatorname{Tr}\left(\frac{e^{-\beta H}}{Z}[H \cdot-H]\right)-T \ln (Z) \stackrel{!}{=} \min .
$$

The proof is given shortly. In Eq. (1) and in the following an index bullet refers to the system studied (at equilibrium), i.e., to the "hard problem" which one likes to learn about by the variational method. $\beta=1 / T, Z=\operatorname{Tr}\left(e^{-\beta H}\right)$. Trial quantities carry no index, so $H$ is the element running through the trial space whose only restrictions are that (a) the spectrum of $H$ is bounded from below and (b) $H$ acts in the Hilbert space of $H$.. The formulation (1) is found, e.g., as Eq. (10.83) in [2] or as Eq. (20.37) in [4]. It is called Gibbs variational principle in [2] and Bogoljubov inequality in [6,4]. There is a natural application to the Heisenberg spin model, where minimizing $\mathcal{V}$ yields the best Curie-Weiss Hamiltonian [4], thereby justifying the mean field procedure.

For the proof of Eq. (1), we claim that one line suffices. It rests on the inequality $-\ln (x) \geqslant 1-x$ and on the irrelevance of operator-ordering under trace, $\operatorname{Tr} \ln (A B)=\operatorname{Tr} \ln (B A)$. With any nonequilibrium statistical operator $\rho$, the line reads

$$
\begin{aligned}
F[\rho] & =\operatorname{Tr}\left(\rho H_{\bullet}\right)+T \operatorname{Tr}(\rho \ln [\rho]) \\
& =F_{\bullet}-T \operatorname{Tr}\left(\rho \ln \left[\frac{1}{\rho} \rho \cdot\right]\right) \geqslant F_{\bullet} .
\end{aligned}
$$

To the left, Eq. (2) starts with the nonequlibrium free energy in $(E-T S)$ form. The knowledge of $\rho$. at equilibrium, $H_{.}=-T \ln \left(\rho_{.} Z_{\bullet}\right)$, is used for the inner equality sign $\left[F_{.}=-T \ln \left(Z_{0}\right)\right]$. Finally, the right end has been simplified using $\operatorname{Tr}(\rho)=\operatorname{Tr}\left(\rho_{\bullet}\right)=1$. In whatsoever nonequilibrium state the system is, it has a statistical operator $\rho$ with the three properties one-trace, Hermiticity, and positivity. Thus [with the properties (a), (b) of $H$ as stated above], its general form is $\rho=e^{-\beta H} / Z$. This makes Eq. (2) to become Eq. (1), Q.E.D.

It is tempting to require that the trial theory be a solvable one (e.g., a free field theory). However, it must not. Imagine there was a small coupling $e$ in the trial theory, and (for simplicity) only one variational parameter $\eta$. Near its minimum, the functional would take the form $\mathcal{V}=a(e)+b(e)$ $\times[\eta-c(e)]^{2}$. Clearly, through perturbative expansion of $V$, the coefficients $a, b, c$ as well as the position $\eta$ of the minimum would be obtained as power series in $e$. The parameter $\eta$ may be chosen to be the coupling $e$ itself.

We now turn to gauge field theory, governing a periodically repeated box of volume $V$ and coupled to a thermal bath at rest with four-velocity $U=(1, \mathbf{0})$. In the variational principle (1) $H$. and $H$ are the Hamiltonians to a Lagrangian $\mathcal{L}$. studied and a trial Lagrangian $\mathcal{L}$, respectively. To count the same number of field degrees of freedom, one may either prepare the physical Hilbert spaces from the outset $[6,8,15]$ or work with extended spaces (corrected by ghosts). In the latter case the two gauge fixings may be different. Adopting general covariant gauges, there is a gauge-fixing parameter $\alpha$. of the theory studied and an $\alpha$ of the trial theory. No final result is allowed to depend on either of them.

The hot gluon system is described by the pure Yang-Mills Lagrangian

$$
\mathcal{L}_{\bullet}=-\frac{1}{4} F_{\cdot \mu \nu}^{a} F_{\cdot}^{\mu \nu a}-\frac{1}{2 \alpha}\left(\partial^{\mu} A_{\mu}^{a}\right)^{2}+i \bar{c}^{a} \partial^{\mu} D_{\mu}^{a b} c^{b},
$$

with $\quad F \cdot{ }_{\cdot \mu \nu}^{a}=\partial_{\mu} A_{\nu}^{a}-\partial_{\nu} A_{\mu}^{a}+g f^{a b c} A_{\mu}^{b} A_{\nu}^{c} \quad$ and $\quad D_{\mu}^{a b}=\delta^{a b} \partial_{\mu}$ $-g f^{a b c} A_{\mu}^{c}$. In the high-temperature limit the one-loop contributions (hard thermal loops) are of relative order unity and 
therefore must be included in the true leading order $[16,17]$. The gluon propagator, resummed this way, may be written as

$$
G^{\mu \nu}(Q)=\frac{\mathrm{A}^{\mu \nu}(Q)}{Q^{2}-M_{t}(Q)}+\frac{\mathrm{B}^{\mu \nu}(Q)}{Q^{2}-M_{l}(Q)}+\alpha \frac{\mathrm{D}^{\mu \nu}(Q)}{Q^{2}},
$$

where $A, B, D$ are members of the symmetric Lorentz matrix basis

$$
\begin{gathered}
\mathrm{A}=g-\mathrm{B}-\mathrm{D}, \quad \mathrm{B}=\frac{V \circ V}{V^{2}}, \\
\mathrm{C}=\frac{Q^{\circ} V+V \circ Q}{\sqrt{2} Q^{2} q}, \quad \mathrm{D}=\frac{Q^{\circ} Q}{Q^{2}},
\end{gathered}
$$

with

$$
V=Q^{2} U-(U Q) Q=\left(-q^{2},-Q_{0} \mathbf{q}\right) .
$$

The orthonormal properties of Eq. (5) are listed in [20]. Note that $A$ and $B$ are projectors:

$$
\mathrm{A}^{\mu \nu}(Q) Q_{\nu}=0, \quad \mathrm{~B}^{\mu \nu}(Q) Q_{\nu}=0 .
$$

In Eq. (4), $M_{t}=\Pi_{t}$ and $M_{l}=\Pi_{l}$ are the well known polarization functions $[21,22]$

$$
\begin{gathered}
\Pi_{t}(Q)=\frac{3}{2} m^{2}-\frac{1}{2} \Pi_{l}(Q), \\
\Pi_{l}(Q)=4 g^{2} N \sum_{P} \Delta_{0}^{-} \Delta_{0}\left[p^{2}-\frac{(\mathbf{p q})^{2}}{q^{2}}\right]
\end{gathered}
$$

with

$$
\begin{gathered}
m^{2}=\frac{g^{2} N T^{2}}{9}, \quad \Delta_{0}=\frac{1}{P^{2}}, \quad \Delta_{0}^{-}=\frac{1}{(Q-P)^{2}}, \\
\sum_{P} \equiv \frac{1}{V} \sum_{\mathbf{p}} T \sum_{n} .
\end{gathered}
$$

For more details on the $\Pi$ 's (especially in our notation) see Appendix $\mathrm{B}$ of [23]. If $V \rightarrow \infty, \Sigma_{P}$ turns into $\int d^{3} p(2 \pi)^{-3} T \Sigma_{n}$. We work with the Matsubara contour: $Q=\left(i \omega_{n}, \mathbf{q}\right), \omega_{n}=2 \pi n T$. The gauge fields are Fourier transformed as

$$
A_{\mu}(x)=\sum_{P} e^{-i P x} A_{\mu}(P), \quad A_{\mu}(P)=\int^{\beta} e^{i P x} A_{\mu}(x)
$$

with $x=(-i \tau, \mathbf{r})$ and $\int^{\beta} \equiv \int_{0}^{\beta} d \tau \int d^{3} r$. To, e.g., check this, the thermal Kronecker symbol

$$
\int^{\beta} e^{i(Q-P) x}=\beta V \delta_{n_{Q}, n_{P}} \delta_{\mathbf{q}, \mathbf{p}} \equiv[Q-P]
$$

is very convenient. In Eq. (4) the bullet on $\alpha$. had been "'forgotten,', because the Green's function (4) will turn out to be that of the trial theory as well.

Were there not the paper [6], a suitable trial Lagrangian could come into mind while contemplating on Eq. (4). Use $n$ free photon Lagrangians (numbered by $a$ ), supply them with variable mass terms such that Eq. (4) is among their propagators, and identify the fields: $A$ in Eq. (3) $\equiv A$ in Eq. (12):

$$
\mathcal{L}=-\frac{1}{4} F^{2}+\frac{1}{2} A(M A)-\frac{1}{2 \alpha}(\partial A)^{2}+i \bar{c} \partial^{2} c
$$

with $F_{\mu \nu}=\partial_{\mu} A_{\nu}-\partial_{\nu} A_{\mu}$. The trivial index $a$ is suppressed here, and

$$
\begin{aligned}
(M A)^{\mu}(x)= & \int_{x^{\prime}}^{\beta} \sum_{Q} e^{-i Q\left(x-x^{\prime}\right)}\left[M_{t}(Q) \mathrm{A}^{\mu \nu}(Q)\right. \\
& \left.+M_{l}(Q) \mathrm{B}^{\mu \nu}(Q)\right] A_{\nu}\left(x^{\prime}\right) .
\end{aligned}
$$

The propagator of Eq. (12) is Eq. (4). But note that we are still free to choose, e.g., constant masses

$$
M_{t}=m_{t}^{2}, \quad M_{l}=m_{l}^{2} \quad(\text { (" } m \text { case') },
$$

or to cover the true leading-order propagators (4) with

$$
M_{t}=\lambda_{t}^{2} \Pi_{t}(Q), \quad M_{l}=\lambda_{l}^{2} \Pi_{l}(Q) \quad(" \lambda \quad \text { case" }) .
$$

Our trial Lagrangian (12) is noninteracting and quadratic in the fields $A$. The gauge-fixing term is necessary, because the mass terms are Abelian gauge invariant. To see this, insert the Fourier transform (10) into Eq. (13) and notice that the gauge variation $\delta A_{\nu}(Q)=-Q_{\nu} \chi(Q)$ drops out due to Eq. (7). Despite these neat properties of the mass term, the longitudinal one makes trouble. By $Q^{\mu} \rightarrow i \partial^{\mu}$ in Eq. (13), and in the $m$ case for simplicity, we may rewrite $A(M A)$ as

$$
A(M A)=A_{\mu}(x)\left[m_{t}^{2} \mathrm{~A}^{\mu \nu}(i \partial)+m_{l}^{2} \mathrm{~B}^{\mu \nu}(i \partial)\right] A_{\nu}(x) .
$$

In passing, Eq. (16) is the Abelian (trivial) case of the Lagrangian considered in [24]. The matrix B (not A) has a denominator containing $Q_{0}: V^{2}=-q^{2} Q^{2} \rightarrow-\Delta \square$. Hence, our trial Lagrangian has arbitrarily high powers in the time derivative. The definition of field momentum densities in higher derivative Lagrangians is a delicate matter, as is the construction of its Hamiltonian. Thus $H$, the trial object, makes the problem. Note that, if working with a constantmass Stueckelberg term $\frac{1}{2} m^{2} A^{\mu} A_{\mu}$ [25], this problem would not yet arise. We leave these difficulties right now, because there is a wonderful way out as detailed in the following subsection.

\section{B. Feynman-Jensen}

To each Lagrangian, Eqs. (3) and (12), there is a partition function which, using functional integrals, is expressed by the actions $S .=-\int^{\beta} \mathcal{L}$. and $S=-\int^{\beta} \mathcal{L}$ :

$$
Z_{\bullet}=\frac{1}{Z_{B}^{\bullet}} \mathcal{N} \int \mathcal{D} A e^{-S .}, \quad Z=\frac{1}{Z_{B}} \mathcal{N} \int \mathcal{D} A e^{-S} .
$$

In Eq. (17), and for the moment, let $\int \mathcal{D} A$ include the ghost field integrations. Equation (17) holds true in Euclidean space [26,21]: $A_{0}$ is a purely imaginary field. The prefactors $Z_{B}^{*}$ and $Z_{B}$, e.g., $Z_{B}=\int \mathcal{D} B \exp \left(-\int^{\beta} B^{2} / 2 \alpha\right)$, occur through the derivation of Eq. (17) while integrating over $\delta(\partial A-B)$ 
with normalized weight. Usually, they are hidden in the functional measure $\mathcal{N}$. But here, the two $\mathcal{N}$ in Eq. (17) are equal and independent of $\alpha$. Nevertheless, they depend on $\beta$ [26]. It might be emphasized that the above functional language can still be applied to the Hamiltonian version $\mathcal{V}[H]$, since the first term of Eq. (1) is $-T \ln (Z)$, and the individual terms in $\left\langle H_{0}-H\right\rangle$ (if known) can be related to Greens functions, which in turn derive from $Z$ (with source terms included).

Hamiltonians can be avoided at all, as we learn in Sec. 3.4 of Feynman's textbook [10] (see also Sec. 8.3,4 there and [11]). Start from $Z$., add the factors $e^{S}$ and $e^{-S}$ under the integral, divide by (and multiply with) $Z$ and define the average $\langle\cdots\rangle$ as given in Eq. (18). Then,

$$
\begin{gathered}
Z_{\bullet}=\frac{Z_{B}}{Z_{B}^{\cdot}} Z\left\langle e^{-(S .-S)}\right\rangle \geqslant \frac{Z_{B}}{Z_{B}^{\cdot}} Z e^{-\langle S \cdot-S\rangle}, \\
\langle\cdots\rangle \equiv \frac{\int \mathcal{D} A e^{-S} \ldots}{\int \mathcal{D} A e^{-S}} .
\end{gathered}
$$

The above inequality is, in the case at hand, the Jensen inequality; see, e.g., [27]. Its simplest version states that $\left\langle e^{-f}\right\rangle \geqslant e^{-\langle f\rangle}$. The proof rests on the convexity of $e^{x}[5,12]$, or, equivalently, on the nice Figure 3.5 of [10]. For convenience we take the logarithm of Eq. (18),

$$
\mathcal{V}[S]=F+T\langle S .-S\rangle-T \ln \left(\frac{Z_{B}}{Z_{B}^{\circ}}\right) \stackrel{!}{=} \min ,
$$

and call Eq. (19) the Feynman-Jensen variational principle. The last term, we come back to shortly, is obviously specific to gauge theory.

In the non-Abelian case, there is a terrible pitfall hidden in Eq. (18). Admittedly, things were written down, to run into it with ease. If one still reads $\int \mathcal{D} A$ to include the ghost field integrations (wrong case), the ghost term would appear in the average $T\langle S .-S\rangle$ and lose the term linear in $A$, hence all $A$ dependence. But if the integration over ghosts is correctly recognized to be the Faddeev-Popov determinant, $D_{\mathrm{FP}}(A)$, it may be included into $S$. as $S_{0}^{\text {no ghosts }}$ $-\ln \left[D_{\mathrm{FP}}(A)\right]$. Now, in the avergage $T\left\langle-\ln \left(D_{\mathrm{FP}}\right)\right\rangle$, even powers of $A$ survive. For the explicit formulation of this see Sec. V. We learn that $i \overline{c c}$, though being Hermitian, must not be viewed as a real number. Hence, in the non-Abelian case one needs to write the action $S$. in Eqs. (18) and (19) as to include $D_{\mathrm{FP}}(A)$, while there will be no ghost field integrations in $\int \mathcal{D} A$.

There is also the Peierls' version $[28,2,4,5]$ of a thermal variational principle. It states that $\operatorname{Tr} e^{-\beta H} \geqslant \Sigma_{n} e^{-\langle n|H| n\rangle}$, rests on Jensen and may be used for another derivation [5] of Eq. (1). Things are closely related. But we have no rigorous answer to the question, whether the two versions (1) and (19) are identical statements-just formulated in different language-or not [see also pt. (3) in Sec. VII]. The Feynman-Jensen variational principle stays useful even at zero temperature. It has been applied at $T=0$ to $\lambda \phi^{4}$ theory without [29] and with gauge sector [18]. Formerly, these efforts had a Hamiltonian formulation, considered even thermal [7]. At finite temperature, but without the exotic last term, Eq. (19) has been recently used to obtain gap equations in lattice $\phi^{4}$ theory [13]. Before, it played a central role in a study of spin models and lattice gauge theory [14].

The role played by the unusual last term in Eq. (19) clears up by combining it with the gauge-fixing terms contained in $S$. and $S$ :

$$
\begin{aligned}
\mathcal{V}_{\text {gauge }} & =\frac{T}{2}\left(\frac{1}{\alpha_{\bullet}}-\frac{1}{\alpha}\right) \int^{\beta}\left\langle(\partial A)^{2}\right\rangle-T \ln \left(\prod_{\mathbf{q}, n}^{\prime} \sqrt{\frac{\alpha}{\alpha_{\bullet}}}\right) \\
& =\left(\frac{\alpha}{\alpha_{\bullet}}-1-\ln \left[\frac{\alpha}{\alpha_{\bullet}}\right]\right) \frac{V}{2} \sum_{Q}^{\prime} .
\end{aligned}
$$

For the logarithm in the first line see (A14); the prime excludes $n=\mathbf{q}=0$. To understand the last term in the second line, remember that $\Sigma_{Q}=(T / V) \Sigma_{n} \Sigma_{\mathbf{q}}$. For the other terms insert Eq. (10) and use

$$
\begin{aligned}
\left\langle A_{\mu}(Q) A_{\nu}(P)\right\rangle & =[Q+P] G_{\mu \nu}(Q) \\
& =[Q+P]\left(\mathrm{A} \Delta_{t}+\mathrm{B} \Delta_{l}+\alpha \mathrm{D} \Delta_{0}\right),
\end{aligned}
$$

where the shorthand notation should be obvious from Eq. (4). Now consider $\alpha$ of the trial Lagrangian to be one of the variational parameters. Clearly, with respect to $\alpha$, Eq. (20) has an extremum at $\alpha=\alpha$., and $\mathcal{V}_{\text {gauge }}$ vanishes at this position. Moreover, it is a minimum, since the blank sum at the end in Eq. (20) is positive (though quartic divergent). By far the best $\alpha$ is $\alpha$. We note three consequences of $\alpha=\alpha$. First, the three terms selected in Eq. (20) may be simultaneously omitted in the sequel. Second, there is still dependence on the now common $\alpha$, as it enters through $\langle\cdots\rangle$ when traced back to the trial propagator (4). Hence, the above selection of $\alpha$-dependent terms was incomplete. But the divergence of the last factor in Eq. (20) helps maintain the conclusion with rigor. Third, with respect to $\alpha$, the variational principle is exhausted, so one should no more think about an "optimal" (common) $\alpha$.

\section{The "even version"}

So far, we were able to circumvent the Hamiltonian dilemma noted at the end of Sec. II A. But in the new version (19) there is again a troubling element, as we become aware of next. Terms odd in the gauge field $A$ (the $A A A$ part of $\mathcal{L}$. in particluar) drop out in $\mathcal{V}$ entirely, because they only enter $\langle S .-S\rangle$ and vanish there, since the average weight is the quadratic trial action. It is as if the three-vertex were taken out from the outset. But a Yang-Mills theory with no three-vertex can never be tested suitably by any trial theory. For more details see the next section.

For the resolution to this puzzle, it appears that the usual philosophy ("improve the trial theory") fails. Also, our trial theory (12) is physically so reasonable: it "must" work. Our way out is to introduce one more version of the variational functional. On one hand this construction, which we call the "even version," is the decisive success in treating gauge fields variationally. On the other hand the idea is rather simple: in general, odd-in- $A$ terms in the action can be avoided from the outset by playing around with the functional integrations over $A$ as follows.

Let us split the action into $S_{\mathbf{.}}=\mathcal{E}+\mathcal{O}$ with $\mathcal{E}$ keeping and $\mathcal{O}$ changing sign under $A \rightarrow-A$. The same decomposition 
can be done with the exponentiated action as $e^{-\mathcal{E}} e^{-\mathcal{O}}=e^{-\mathcal{E}} \cosh (\mathcal{O})-e^{-\mathcal{E}} \sinh (\mathcal{O})$. Since the second term drops out under the functional integrations over the gauge field $A$, we may write

$$
\int \mathcal{D} A e^{-S} \cdot=\int \mathcal{D} A e^{-\mathcal{E}+\ln [\cosh (\mathcal{O})]}
$$

The new exponent, which we call $-S .$. , is an even functional of $A$. Since the above steps precede the use of Jensen's inequality, quite a new functional $\mathcal{V}$ arises:

$$
\mathcal{V}[S]=F+T\left\langle S_{. .}-S\right\rangle \stackrel{!}{=} \min \quad \text { with } \quad S . .=\mathcal{E}-\ln [\cosh (\mathcal{O})] .
$$

In Eq. (23) $\alpha=\alpha$. is understood, i.e., the logarithm of $Z_{B}$ 's is omitted together with the gauge-fixing terms in $\mathcal{E}$ and $S$.

Once there are only even terms in the theory studied, the quadratic trial theory has a good chance to reproduce the leading-order perturbative results. We shall show in Secs. IV and $\mathrm{V}$ that the "even version" works that way, indeed. There, the Faddeev-Popov determinant (depending on $A$ in Sec. V, but not in Sec. IV) is part of $S$. and hence subject of the above "even' -ing procedure.

\section{TRIAL AND ERROR}

In this short section we step back to the insufficient Feynman-Jensen formulation (19) to see which way it goes wrong, to introduce some basic integrals and for a first run through the necessary algebra in the simplest case. For simplicity, let us even omit the Faddeev-Popov term [i.e., run into the pitfall noticed below Eq. (19)]. It is not (solely) responsible for the defect, as we shall remark at the end of this section.

Using $\alpha=\alpha$. as reasoned below Eq. (21), the functional reads $\mathcal{V}=F+T\langle S .-S\rangle$. In the difference $S .-S$ $=-\int \beta(\mathcal{L} .-\mathcal{L})$ the terms odd in the gauge field $A$ vanish under the average $\langle\cdots\rangle$. Others cancel. The only two surviving terms are

$$
\begin{aligned}
T\langle S .-S\rangle & =V \frac{1}{2}\left\langle A^{a}\left(M A^{a}\right)\right\rangle+V \frac{g^{2}}{4} f^{a b c} f^{a r s}\left\langle A_{\mu}^{b} A_{\nu}^{c} A^{\mu r} A^{\nu s}\right\rangle \\
& \equiv \mathcal{V}_{M}+\mathcal{V}_{A A A A},
\end{aligned}
$$

where $\int^{\beta}$ has reduced to $\beta V$ due to spacetime independence of the averages. The first term, $\mathcal{V}_{M}$ with $M$ from Eq. (13), is readily evaluated by using Eqs. (10) and (21) and the trace relation $\left(\left[M_{t} \mathrm{~A}+M_{l} \mathrm{~B}\right] G\right)_{\mu}^{\mu}=2 M_{t} \Delta_{t}+M_{l} \Delta_{l}$ :

$$
\mathcal{V}_{M}=n V T^{4}\left(-L_{t}-\frac{1}{2} L_{l}\right)
$$

$L_{t, l}$ are two sums out of the collection

$$
\begin{gathered}
J_{t, l}=-\beta^{2} \sum_{P} \Delta_{t, l}, \quad L_{t, l}=-\beta^{4} \sum_{P} P^{2}\left(\Delta_{t, l}-\Delta_{0}\right), \\
Y_{t, l}=\beta^{2} \sum_{P} p^{2} \Delta_{0} \Delta_{t, l}
\end{gathered}
$$

with $\Delta_{t, l}=1 /\left[P^{2}-M_{t, l}(P)\right]$ and $\Delta_{0}=1 / P^{2}$. The prefactor $n$ in Eq. (25) comes from the trivial sum over the color index.

The treatment of $\mathcal{V}_{A A A A}$ starts with the Wick decomposition [6] of the average into three pairs with partners

$$
\left\langle A_{\mu}^{a}(x) A_{\nu}^{b}(x)\right\rangle=\delta^{a b} \sum_{P} G_{\mu \nu}(P)=\delta^{a b} T^{2} \frac{1}{3}\left(u_{\mu \nu} r+v_{\mu \nu} s\right) .
$$

The first equality in Eq. (27) derives with Eqs. (10) and (21). The second one arises after integration over the directions of p. As the propagators $\Delta_{t, l}$ are rotationally invariant [even in the $\lambda$ case (15)], this angular integration amounts to the replacements $\mathrm{A} \rightarrow-\frac{2}{3} u, \quad \mathrm{~B} \rightarrow-\frac{1}{3} u-\frac{1}{3} p^{2} \Delta_{0} v$, and $\mathrm{D} \rightarrow \frac{1}{3}(v-u)+\frac{1}{3} p^{2} \Delta_{0} v$ with the Lorentz matrices $u_{\mu \nu}, v_{\mu \nu}$ given by $U_{\mu} U_{\nu}-g_{\mu \nu}$ and $4 U_{\mu} U_{\nu}-g_{\mu \nu}$, respectively. For the sums $r$ and $s$ see Eq. (29) below. Using the first equation (27) and with $f^{a b c} f^{a b c}=n N$ one derives the first line of Eq. (28). Exploiting the $u-v$ version, one arrives at the second one:

$$
\begin{aligned}
\mathcal{V}_{A A A A} & =n V \frac{g^{2} N}{4}\left(\left[\sum_{P} G_{\mu}^{\mu}(P)\right]^{2}-\sum_{P} G_{\mu \nu}(P) \sum_{Q} G^{\mu \nu}(Q)\right) \\
& =n V T^{4} \frac{g^{2} N}{6}(r+s)(r-2 s) .
\end{aligned}
$$

The objects $r, s$ in Eqs. (27) and (28) are given by

$$
r=2 J_{t}+J_{l}+\alpha J_{0}, \quad s=-Y_{l}-\alpha J_{0}+\alpha Y_{0},
$$

where $J_{0}$ and $Y_{0}$ are the sums of Eq. (26) taken at vanishing mass.

The last term of $\mathcal{V}$ to be evaluated is the trial free energy $F=-T \ln (Z)$. First of all, since colors do not mix, $Z$ is an $n$ fold product,

$$
F=n F_{\text {colorless }}=-n T \ln \left(Z_{\text {colorless }}\right)=n V T^{4}\left(-2 I_{t}-I_{l}+I_{0}\right),
$$

and the colorless partition function is identical with that of scalar ED, see Sec. IV, if omitting the factor due to the scalars. In the formula (17) for $Z$ (read colorless and Euclidean), there are three unknown flying objects: $\mathcal{N}, \int \mathcal{D} A$, and $\int \mathcal{D} B$. This is not a shame if $Z$ is used exclusively as a generating functional. But here we need $Z$ as a precise number. The trouble [26] with the normalization factor $\mathcal{N}$ is proportional to the care of its treatment. We make efforts in Appendix A to write down at least (if not to derive) this factor $\mathcal{N}$. Here we see from Eq. (A20) that $F$ indeed splits up into the terms in the right-hand side of Eq. (30). With Eq. (A3), we obtain

$$
I_{l}=\frac{1}{2 V T^{3}} \sum_{\mathbf{p}}\left[\ln \left(-T^{2} \Delta_{l}\left(P_{0}=0, \mathbf{p}\right)\right)+\sum_{n}{ }^{\prime} \ln \left(P_{0}^{2} \Delta_{l}(P)\right)\right],
$$

where the prime excludes $n=0$. The index $l$ may be replaced by $t$ or by 0 (then referring to zero mass). The expression (31) sticks with this awkward form as long as the $\lambda$ case (15) is included. But by differentiation with respect to $\lambda_{l}$ we may write 


$$
-\lambda_{l} \partial_{\lambda_{l}} I_{l}=L_{l} \quad \text { or } \quad I_{l}=I_{0}-\int_{0}^{\lambda_{l}} d \lambda \frac{1}{\lambda} L_{l}\left(\lambda_{l}=\lambda\right),
$$

the right half being equivalent to a coupling constant integration. In the $m$ case the above relation reads $-m \partial_{m} I=L$.

The sums $I, J$ to $Y$ are divergent, and one has to keep track of variational-parameter dependences while renormalizing [6]. To study this in simple terms (and for the rest of this section) we turn to constant masses by Eq. (14). In this case the frequency sum in Eq. (31) can be done [21]. Using Eq. (A7) and going to the infinite volume limit, one obtains

$$
\begin{gathered}
I_{l}=-\frac{1}{2 \pi^{2}} \int_{0}^{\infty} d x x^{2}\left[\frac{1}{2} \sqrt{x^{2}+\varepsilon_{l}^{2}}+\ln \left(1-e^{-\sqrt{x^{2}+\varepsilon_{l}^{2}}}\right)\right], \\
\varepsilon_{l} \equiv \beta m_{l} .
\end{gathered}
$$

Furthermore, $L_{l}=\varepsilon_{l}^{2} J_{l}$. The sum $J$ becomes

$$
J_{l}=\frac{1}{2 \pi^{2}} \int_{0}^{\infty} d x \frac{x^{2}}{\sqrt{x^{2}+\varepsilon_{l}^{2}}}\left[\frac{1}{2}+\frac{1}{e^{\sqrt{x^{2}+\varepsilon_{l}^{2}}}-1}\right]
$$

with clearly the $\frac{1}{2}$ term being UV divergent as in Eq. (33). Even after subtracting zero-point energies by hand (which the functional integral does not know of), $I_{l} \rightarrow I_{l}+\left(1 / 4 \pi^{2}\right) \int_{0}^{\infty} d x x^{3} \equiv I_{l}^{\text {sub }}$ there remains a singular integral depending on the variational parameter $\varepsilon_{l}$. On the other hand, in a low-order perturbative treatment, such terms can be addressed as zero-temperature renormalization [16,23] and omitted entirely. As we like to reproduce these results only, the omission should be allowed here as well. Consider, for example, the combination $-I_{l}^{\text {sub }}-\frac{1}{2} L_{l}$, which occurs in $F+V_{M}$, and supply $p$ with an UV cutoff $\Lambda$ :

$$
\begin{aligned}
& {\left[-I_{l}^{\mathrm{sub}}-\frac{1}{2} L_{l}\right]_{1 / 2 \text { term }}} \\
& \quad=\frac{1}{4 \pi^{2}} \int_{0}^{\Lambda / T} d x\left(\sqrt{x^{2}+\varepsilon_{l}^{2}}-x-\frac{\varepsilon_{l}^{2}}{2 \sqrt{x^{2}+\varepsilon_{l}^{2}}}\right) \\
& \quad=\frac{\varepsilon_{l}^{4}}{32 \pi^{2}} \ln \left(\frac{\Lambda}{T \varepsilon_{l}}\right)+O\left(\varepsilon_{l}^{4}\right) .
\end{aligned}
$$

Since we expect $\varepsilon_{l} \sim g$, such terms are irrelevant in $\mathcal{V}$ up to $g^{3}$. In the sequel we shall trust in the above arguments and omit the $\frac{1}{2}$ terms entirely.

Deleting the divergent pieces this way [in, e.g., Eqs. (33) and (34)], I, $J, Y$ become well defined integrals whose asymptotic series are known [30]:

$$
\begin{gathered}
I=\frac{\pi^{2}}{90}-\frac{\varepsilon^{2}}{24}+\frac{\varepsilon^{3}}{12 \pi}+\frac{\varepsilon^{4}}{32 \pi^{2}} \ln (\varepsilon)-\frac{c \varepsilon^{4}}{64 \pi^{2}}+O\left(\varepsilon^{6}\right), \\
J=\frac{1}{12}-\frac{\varepsilon}{4 \pi}+\cdots=-\frac{1}{\varepsilon} \partial_{\varepsilon} I=\frac{1}{\varepsilon^{2}} L,
\end{gathered}
$$

$$
Y=\frac{1}{8}-\frac{\varepsilon}{4 \pi}+\cdots=\frac{3}{\varepsilon^{2}}\left(I_{0}-I\right)
$$

with $\varepsilon$ one of $\varepsilon_{t, l}=\beta m_{t, l}, c=\frac{3}{2}+2 \ln (4 \pi)-2 \gamma$ and $\gamma$ the Euler constant. In the massless limit, the free energy (30) is now recognized to be $n$ times that of ordinary blackbody radiation.

The contributions to $\mathcal{V}$ are now added up as $F+\mathcal{V}_{M}+\mathcal{V}_{A A A A}$ and filled with details:

$$
\begin{aligned}
\mathcal{V}= & n V T^{4}\left[-2 I_{t}-\varepsilon_{t}^{2} J_{t}+\frac{1}{2}\left[-2 I_{l}-\varepsilon_{l}^{2} J_{l}\right]+I_{0}\right. \\
& \left.+\frac{g^{2} N}{6}\left(2 J_{t}+J_{l}-Y_{l}+\frac{\alpha}{8}\right)\left(2 J_{t}+J_{l}+2 Y_{l}\right)\right] \\
= & \operatorname{const}+\frac{n V T^{4}}{4 \pi}\left(\frac{\varepsilon_{t}^{3}}{3}-\varepsilon_{t} g^{2} N \frac{5+\alpha}{24}+\frac{\varepsilon_{l}^{3}}{6}\right. \\
& \left.-\varepsilon_{l} g^{2} N \frac{1+\alpha}{16}+\cdots\right) .
\end{aligned}
$$

There it is, the announced wrong result: $\mathcal{V}$ depends on $\alpha$. Nevertheless, the structure is appealing: the parameters $\varepsilon_{t}$ and $\varepsilon_{l}$ do not mix, the only extremum is a minimum, and its position has the right order $g^{2} N$ of magnitude. But, apart from this, the minimum positions $\varepsilon_{t}^{2}=g^{2} N(5+\alpha) / 24$ and $\varepsilon_{l}^{2}=g^{2} N(1+\alpha) / 8$ give no sense: which $\alpha$ ? Including the FP term, with the means worked out in Sec. V, does not help us out of this dilemma, because it only leads to minor changes. To be specific, in Eq. (40) $5+\alpha$ becomes $6+\alpha$ and $1+\alpha$ turns into $(2+3 \alpha) / 3$.

\section{SCALAR ELECTRODYNAMICS}

For a first application of the "even" functional (23), we appreciate scalar ED as a suitable example. Remember that this system is an ideal toy model [19] to the gluon plasma, with view to the identical diagram structure, the need of resummation as well as to its physical gross features. The Lagrangian, to be studied, is given by

$$
\mathcal{L}_{.}=\left(D^{\mu} \phi\right)^{*} D_{\mu} \phi-\frac{1}{4} F^{2}-\frac{1}{2 \alpha .}(\partial A)^{2}
$$

with $D_{\mu}=\partial_{\mu}-i g A_{\mu}$ and $F_{\mu \nu}=\partial_{\mu} A_{\nu}-\partial_{\nu} A_{\nu}$. By again identifying the fields (here: $\phi$ and $A$ ), the trial Lagrangian reads

$$
\mathcal{L}=\left(\partial^{\mu} \phi\right)^{*} \partial_{\mu} \phi-\frac{1}{4} F^{2}-\frac{1}{2 \alpha}(\partial A)^{2}+\frac{1}{2} A(M A)-m_{s}^{2} \phi^{*} \phi .
$$

Its propagators are Eq. (4) and $1 /\left[m_{s}^{2}-Q^{2}\right]$ for photons and scalars, respectively. Here we concentrate on the spectum of real excitations. Hence, the mass matrix $M$ is that of the $\lambda$ case (15). The variational parameters in the above trial theory are $\lambda_{t}, \lambda_{l}$ and the scalar mass $m_{s}$. The Lagrangian (42) turns into the effective Lagrangian (at order $g^{2}$ ) of hot scalar ED [19] at the values $\lambda_{t}=\lambda_{l}=1$ and $m_{s}^{2}=g^{2} T^{2} / 4$. So, within $O\left(g^{2}\right)$, the parameter space includes the exact answer (to be derived by variation). Note that both original and trial 
theory are invariant under regauging the photon field by $\delta A=-\partial \Lambda$. By definition, the decoupling ghost terms are kept apart from the above Lagrangians. But the FaddeevPopov compensation must be taken into account in the partition function either by ghosts or as a determinant.

\section{A. The "even" functional of scalar ED}

Recalling Sec. II C, the partition function of scalar ED may be written as

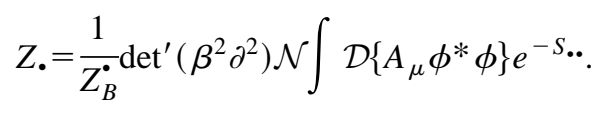

The prime on the Faddeev-Popov determinant excludes the zero eigenvalue (see also Appendix A). To specify $\mathcal{E}$ and $\mathcal{O}$ in Eq. (23), in the case at hand, we read off from Eq. (41) that

$$
\mathcal{O}=-\int^{\beta} \mathcal{L}_{1} \quad \text { with } \quad \mathcal{L}_{1}=i g A^{\mu} \phi^{*} \partial_{\mu} \phi-i g\left(\partial_{\mu} \phi^{*}\right) A^{\mu} \phi,
$$

while $-\int^{\beta} \mathcal{L}_{2}$ with $\mathcal{L}_{2}=g^{2} A^{\mu} A_{\mu} \phi^{*} \phi$ is part of $\mathcal{E}$ together with the quadratic terms in Eq. (41). The index on $\mathcal{L}$ refers to $g$ powers. With $\alpha=\alpha_{\text {e }}$, as required for Eq. (23) to be valid, we may thus write the "even" functional as

$$
\begin{aligned}
\mathcal{V}= & F+T\left\langle\int \frac{\beta}{2} A(M A)-m_{s}^{2}{ }^{\beta} \phi^{*} \phi-\int^{\beta} \mathcal{L}_{2}\right. \\
& \left.-\ln \left[\cosh \left(\int^{\beta} \mathcal{L}_{1}\right)\right]\right\rangle,
\end{aligned}
$$

where $F=-T \ln (Z)$, and the trial partition function $Z$ is given by Eq. (43) with all bullets stripped off there.

There is a high (but probably inevitable) price to be paid for the physical consistency reached with the above formulation: it obviously contains fields in arbitrary high powers (instead of only quartic). For the explicit evaluation of Eq. (45) one is, apparently, forced again into a perturbative expansion, namely that of the logarithmic term $\left(\ln [\cosh (x)]=x^{2} / 2-x^{4} / 12+x^{6} / 45-\cdots\right)$. But note, at least, that this expansion looks much simpler than diagrammatic thermodynamics: here the seagull vertex does not occur in higher powers. If, for any reason, terms of order $g^{4}$ may be neglected, then the functional simplifies to

$$
\begin{aligned}
\mathcal{V}_{\text {trunc }}= & F+T\left\langle\iint^{\beta} \frac{1}{2} A(M A)-m_{s}^{2} \int^{\beta} \phi^{*} \phi-\int^{\beta} \mathcal{L}_{2}\right. \\
& \left.-\frac{1}{2}\left(\int^{\beta} \mathcal{L}_{1}\right)^{2}\right\rangle .
\end{aligned}
$$

In the following, while demonstrating the value of Eq. (45), we shall in fact restrict to the truncated version Eq. (46).

\section{B. Evaluation of $V_{\text {trunc }}$}

Let us group the above five contributions into "bare" and interaction terms:

$$
\begin{gathered}
\mathcal{V}_{\text {trunc }}=\mathcal{V}_{0}+\mathcal{V}_{\text {int }} \quad \text { with } \quad \mathcal{V}_{0}=F+\mathcal{V}_{M}+\mathcal{V}_{m_{s}^{2}} \\
\mathcal{V}_{\text {int }}=\mathcal{V}_{A A \phi \phi}+\mathcal{V}_{\text {square }}
\end{gathered}
$$

For $V_{0}$ we are well prepared from Sec. III: strip off the color factor $n$ from Eqs. (25) and (30). Of course, with a view to Eq. (A20), the free energy $V T^{4}\left(-2 I_{s}\right)$ of the scalars has to be added now. As the scalars have constant mass, $I_{s}$ is given by Eq. (33) with index $l$ replaced by $s$. For $\mathcal{V}_{m_{s}^{2}}$ note that the average $\left\langle\phi^{*} \phi\right\rangle$ equals $\Sigma_{P} S(P)$ with the scalar propagator given by $S(P)=-1 /\left(P^{2}-m_{s}^{2}\right) \equiv-\Delta_{s}$. Thus, in particular

$$
\begin{gathered}
\mathcal{V}_{m_{s}^{2}}=-V m_{s}^{2}\left\langle\phi^{*} \phi\right\rangle=-V T^{4} L_{s}, \quad L_{s}=\varepsilon_{s}^{2} J_{s}, \\
\varepsilon_{s} \equiv \beta m_{s}, \quad J_{s}=-\beta^{2} \sum \Delta_{s},
\end{gathered}
$$

and in total

$$
\mathcal{V}_{0}=V T^{4}\left(-2 I_{t}-L_{t}-I_{l}-\frac{1}{2} L_{l}+I_{0}-2 I_{s}-L_{s}\right)
$$

Among the interaction terms, one is pretty simple:

$$
\begin{aligned}
\mathcal{V}_{A A \phi \phi} & =-V g^{2}\left\langle\phi^{*} \phi\right\rangle\left\langle A^{\mu} A_{\mu}\right\rangle \\
& =V T^{4} g^{2}\left(2 J_{t}+J_{l}\right) J_{s}+V T^{4} g^{2} \alpha J_{0} J_{s}=F^{\infty}
\end{aligned}
$$

Of course, $J_{0}=1 / 12$ even in the $\lambda$ case. To the right in Eq. (50), we have noted that $\mathcal{V}_{A A \phi \phi}$ precisely equals the perturbative free energy contribution from the diagram $\infty$ (one loop scalar, one photonic). But here the lines represent massive propagators, making $\infty$ depending on variational parameters. One may speculate that the remaining term $\mathcal{V}_{\text {square }}$ could correspond to the diagram $\ominus$ (the inner line photonic). This is indeed the case; see Eq. (51) below. Classes of diagrams were whisked away in the treatment of Sec. III.

The first steps in treating $\mathcal{V}_{\text {square }}$ are straightforward: Fourier transform all fields, Wick decompose (the $\bigcirc-\bigcirc$ diagram drops out due to odd summand), and use Eq. (21). One obtains

$$
\begin{aligned}
\mathcal{V}_{\text {square }} & =-V \frac{1}{2} g^{2} \sum_{Q} \sum_{P}(2 P-Q)^{\mu} G_{\mu \nu}(Q)(2 P-Q)^{\nu} \Delta_{s} \Delta_{s}^{-} \\
& =F^{\ominus}
\end{aligned}
$$

with $\Delta_{s}^{-}=1 /\left[(Q-P)^{2}-m_{s}^{2}\right]$. There is gauge-fixing dependence in $\mathcal{V}_{\text {square }}$ through the propagator $G$ from Eq. (4). After some algebra one obtains that

$$
\mathcal{V}_{\text {square }}-\mathcal{V}_{\text {square }}^{(\alpha=0)}=-V T^{4} g^{2} \alpha J_{0} J_{s},
$$

which cancels the $\alpha$ term of Eq. (50). Thus, in the case of scalar ED and in its truncated "even" functional, there is no gauge-fixing dependence. This is true for all values of our variational parameters and for any mass matrix version.

In Eq. (51) at $\alpha=0$ terms with $Q^{\mu}$ or $Q^{\nu}$ are projected out. The remaining sandwiches are

$$
P A P=-\left[\mathbf{p}^{2}-(\mathbf{p q})^{2} / q^{2}\right]
$$

and 


$$
P \mathrm{~B} P=P^{2}-(P Q)^{2} / Q^{2}-P \mathrm{~A} P .
$$

Expecting the structure (40), at least the terms linear in $\lambda_{t}$, $\lambda_{l}, m_{s}$ must be detailed. We therefore form differences as, e.g., $\Delta_{t l} \equiv \Delta_{t}-\Delta_{l}$, add Eq. (51) to Eq. (50), write $J_{t 0} \equiv J_{t}-J_{0}$, etc., and split $\mathcal{V}_{\text {int }}$ into a constant $\left(v_{0}\right)$, terms linear in such differences $\left(v_{1}\right)$ and the rest $\left(v_{2}\right)$, which is certainly of higher order. Then, some terms of $v_{1}$ [easily identified in Eq. (56) below] are regrouped into $v_{2}$, because they are of higher order for other reasons. Note that

$$
\Sigma_{P}\left[4 P^{2} Q^{2}-4(P Q)^{2}+Q^{4}\right] /\left[Q^{2} P^{2}(Q-P)^{2}\right]=2 \Sigma_{P} 1 / P^{2} .
$$

We obtain:

$$
\mathcal{V}_{\mathrm{int}}=V g^{2}\left(v_{0}+v_{1}+v_{2}\right)
$$

with

$$
v_{0}=\sum_{Q} \sum_{P} \frac{1}{Q^{2} P^{2}}\left(3-2 \frac{Q^{2} P^{2}-(Q P)^{2}}{Q^{2}(Q-P)^{2}}\right)=\frac{5 T^{4}}{288},
$$

In each term of Eq. (56) the argument of the first propagator is $Q$, and $P$ that of the second. To understand why the last two terms of Eq. (56) are less than $O(g)$, the first one can be rewritten as $-2 m_{s}^{2} \Sigma_{Q, P} \Delta_{l} \Delta_{s} \Delta_{s}^{-}$plus some products of differences. But for the last term in Eq. (56) only a detailed analysis (of the type done in Appendix B) reveals its order $T^{4} g^{2} \ln (g)$ of magnitude. Such terms are known to occur in the perturbation expansion of the free energy [31].

Up to order $g^{3}$ in $\mathcal{V}$ (or order $g$ in $v_{1}$ ) only the line (55) needs further study. Note at first that, formally, the expression (8) (at $N=1$ here) appears in this line. So, the machinery "knows" of the leading-order longitudinal polarization function. There are two ways to evaluate $\Sigma_{Q} \Delta_{l t} \Pi_{l}$ (for later use, we detail both). First, one may cancel $\Pi$ functions with those in the trial propagators (using $\Pi_{l}=3 m^{2}-2 \Pi_{t}$ ), write $v_{1}$ in terms of basic integrals as

$$
\sum_{Q} \Delta_{l t} \Pi_{l}=-T^{4}\left(\frac{2}{\lambda_{t}^{2}} L_{t}+\frac{1}{\lambda_{l}^{2}} L_{l}-\frac{g^{2}}{3} J_{t}\right)
$$

and proceed with expanding the latter (see below). Note that these cancellations are possible in the $\lambda$ case only. The alternative second way is by far the easier and more enlightening one. As is basic to the dimensional reduction method [32-34] and to various related thermodynamic calculations (e.g., [35,31]), a frequency sum may be occasionally reduced to its $Q_{0}=0$ term. This step, if valid $[19,36]$, rests on the structure of a massive propagator $\Delta(Q)$ and usually prepares its soft part while contributions from nonzero hard frequencies are of higher order. Of course, there must be no hard part in a sum under such study. In fact, Eq. (57) is an ideal example for the above. Moreover, at $Q_{0}=0$ the polarization functions $\Pi_{l}$ and $\Pi_{t}$ reduce to constants, namely $3 \mathrm{~m}^{2}$ and 0 , respectively:

$$
\begin{aligned}
\sum_{Q} \Delta_{l t} \Pi_{l} & =3 m^{2} \frac{T}{2 \pi^{2}} \int_{0}^{\infty} d q q^{2}\left(\frac{1}{q^{2}}-\frac{1}{q^{2}+\lambda_{l}^{2} 3 m^{2}}\right) \\
& =\frac{T^{4} g^{2}}{12 \pi}\left(\frac{g \lambda_{l}}{\sqrt{3}}\right) .
\end{aligned}
$$

Dependence on $\lambda_{t}$ has dropped out, with the reason readily detected in the vanishing factor $\Pi_{t}$ of $\lambda_{t}^{2}$ i.e., in the absence of a (squared) magnetic mass at order $g^{2}$. In the same manner the soft parts of $J$ integrals are obtained:

$$
J_{l}=J_{0}-\frac{1}{4 \pi} \frac{g \lambda_{l}}{\sqrt{3}}+\cdots, \quad J_{t}=J_{0}+0+\cdots
$$

with again no dependence on $\lambda_{t}$ for the same reason.

To complete the evaluation, note that the scalar contributions $J_{s}, L_{s}, I_{s}$ are $m$-case objects, hence their expansions are given by Eqs. (36) and (37) with $\varepsilon=\varepsilon_{s}=\beta m_{s}$. For the remaining $\lambda$-case integrals in $\mathcal{V}_{0}$, Eq. (49), apparently, the sums $L_{t}$ and $L_{l}$ are still to be studied separately. For this somewhat delicate task see Appendix B. As a result, both $L$ start with a $g^{2} \lambda^{2}$ term whose prefactor $\kappa$ diverges logarithmically. From Eqs. (B5) to (B7),

$$
\begin{gathered}
L_{l}=2 \kappa \frac{g^{2} \lambda_{l}^{2}}{3}-\frac{1}{4 \pi}\left(\frac{g \lambda_{l}}{\sqrt{3}}\right)^{3}+\cdots, \\
L_{t}=\left(\frac{1}{24}-\kappa\right) \frac{g^{2} \lambda_{t}^{2}}{3}+0+\cdots .
\end{gathered}
$$

Now note that the singular piece $\kappa$ drops out in $\mathcal{V}_{0}$, because there $L$ appears in the combination $2 I+L=2 I_{0}+\{1$ $\left.-2 \int_{0}^{\lambda} d \lambda(1 / \lambda)\right\} L$, see Eq. (32), and the curly brackets is a 
projector: \{\}$\lambda^{2}=0$. One might ask for the fate of the singular terms in Eq. (57). They drop out there by cancellation, and Eq. (58) derives again.

\section{Minimizing $\mathcal{V}_{\text {trunc }}$ at order $g^{3}$}

In the preceding subsection, the expansions were driven just as far as to allow for writing down the functional $\mathcal{V}$ up to third order in the coupling $g$. Of course, as in Sec. III, we anticipate that the solutions to $m_{s}$ and $\lambda_{t, l}$ will be $O(g T)$ and order 1 in magnitude, respectively. By combining the details of the preceding subsection one obtains

$$
\begin{aligned}
\mathcal{V}^{\operatorname{tog}^{3}=} & V T^{4}\left(-2 \frac{\pi^{2}}{45}+\frac{5 g^{2}}{288}+\frac{g^{3}}{24 \pi \sqrt{3}}\left[\frac{\lambda_{l}^{3}}{3}-\lambda_{l}\right]\right. \\
& \left.+\frac{1}{12 \pi}\left[\left(\beta m_{s}\right)^{3}-\frac{3 g^{2}}{4} \beta m_{s}\right]\right) .
\end{aligned}
$$

It still has the structure of Eq. (40). The variational parameters do not couple, which is specific to the order considered. The absence of any dependence on $\lambda_{t}$ was already understood, although merely technically (see also Sec. VI). The above $\mathcal{V}$, when plotted over the $\lambda_{l}-\lambda_{t}-$ plane, has the form of a long gutter. The resolution of this defect is deferred to Sec. IV D.

Minimizing Eq. (61) with respect to $m_{s}$ and $\lambda_{l}$ gives the values

$$
m_{s}^{\min }=\frac{1}{2} g T, \quad \lambda_{l}^{\min }=1,
$$

as expected. We immediately look for the value of the above $V$ taken at these parameters, which is the height of the bottom of the gutter:

$$
V^{\min }=V T^{4}\left(-2 \frac{\pi^{2}}{45}+\frac{5 g^{2}}{288}-\frac{g^{3}}{12 \pi}\left[\frac{1}{3 \sqrt{3}}+\frac{1}{4}\right]\right),
$$

with the last term in the square bracket being due to the scalars. The minimum perfectly agrees with the perturbative free energy up to $g^{3}$. The $g^{3}$ term, the correlation energy, was given by Kalashnikov and Klimov [37] [Eq. (19) there, taken at $\lambda=\mu=0$ and $e=g]$. In summary, for scalar electrodynamics and up to the third $g$ power, the "even'" variational functional has all required properties, namely gaugefixing independence, the right minimal value, and (apart from degeneracy) the right minimum position.

\section{Solution to the gutter problem}

The missing dependence on $\lambda_{t}$ in Eq. (61) is, as already noticed, an artifact of the restriction to order $g^{3}$ of the functional. The problem merely is how to go one order higher within the expansions so far developed. First of all, we notice that $g^{4}$ terms are allowed within the truncated functional, although the neglected next term of $\ln [\cosh (x)]$ does contribute at order $g^{4}$ too. However, the latter is a constant at this order; variational parameters appear at $g^{5}$.
Let us try to avoid expansions, and let the collection of all terms containing $\lambda_{t}$ be denoted by $\mathcal{V}_{\text {trunc }}^{(t)}$ Up to an additive constant, it may be written as

$$
\mathcal{V}_{\text {trunc }}^{(t)}=V T^{4} \mathcal{U}_{t}+V g^{2} v_{2}^{(t)}
$$

with

$$
\mathcal{U}_{t}=-L_{t}+2 \int_{0}^{\lambda_{t}} d \lambda \frac{1}{\lambda} L_{t}\left(\lambda_{t}=\lambda\right)+\frac{1}{\lambda_{t}^{2}} L_{t} .
$$

Here, $v_{2}^{(t)}$ is made up of the first two terms in Eq. (56), but leave $v_{2}^{(t)}$ aside for a moment. Then, the minimum condition may be given the form of a product

$$
0=\partial_{\lambda_{t}} \mathcal{U}_{t}=\left(\frac{2}{\lambda_{t}} L_{t}-\partial_{\lambda_{t}} L_{t}\right)\left(1-\frac{1}{\lambda_{t}^{2}}\right)
$$

with the first factor "unknown," but the second reaching zero at $\lambda_{t}^{2}=1$ as desired. To be sure that this zero corresponds to a minimum, the first factor must be shown to be positive. We shall do so at the end of Appendix B. There, the first factor is also seen to be of order $g^{4}$ and to vary as $\lambda_{t}^{3}$ for small $g$, see (B11). Hence, $\mathcal{U}_{t}$ has a Higgs-type shape $\mathcal{U}_{t} \sim$ const $-g^{4} \lambda_{t}^{2}+\frac{1}{2} g^{4} \lambda_{t}^{4}+\cdots$ with a maximum at the origin. The curvature of the gutter sets in one order higher, indeed. A plot of $\mathcal{V}$ now merely looks like a long bathtub.

The above construction only works if the correction $v_{2}^{(t)}$ remains below the order $O\left(g^{2}\right)$. Its first term is the first in Eq. (56) and is of order $T^{4} g\left(J_{t}-J_{0}\right)$ in magnitude. With a view to Eq. (59) it is indeed below $g^{2}$. For the second contribution [the second in Eq. (56) but with $\Delta_{t 0}$ in place of $\left.\Delta_{t l}\right]$ we need a bit of calculation. Both sums may be considered "soft," i.e., $n(x) \rightarrow T / x$ is allowed, thereby preparing the contribution of interest. All propagators are represented spectrally. For the two frequency sums, Eq. (6.6) of [23] is used repeatedly. The result is a three-momentum double integral over (among other factors) $\int d x(1 / x)\left[\rho_{t}^{(\lambda)}(x, q)\right.$ $\left.-\rho^{(0)}(x, q)\right]$. But, due to the sum rule (C6), this factor vanishes. Q.E.D.

\section{YANG-MILLS FIELDS (THE GLUON PLASMA)}

For treating the non-Abelian theory (3) in its "even version," we use Sec. IV as a guideline. Hence, first of all, we strip off the ghost terms from $\mathcal{L}$. $\mathcal{L}$ and introduce the index " "no", for such reduced Lagrangians:

$$
S_{\bullet}^{\mathrm{no}}=-\int^{\beta} \mathcal{L}_{\bullet}^{\mathrm{no}}=-\int^{\beta}\left(\mathcal{L}_{0}+\mathcal{L}_{1}+\mathcal{L}_{2}\right)
$$

with

$$
\begin{gathered}
\mathcal{L}_{1}=-g\left(\partial_{\mu} A_{\nu}^{a}\right) f^{a b c} A^{\mu b} A^{\nu c}, \\
\mathcal{L}_{2}=-\frac{1}{4} g^{2} f^{a b c} f^{a r s} A_{\mu}^{b} A_{\nu}^{c} A^{\mu r} A^{\nu s} .
\end{gathered}
$$

Here, $\mathcal{L}_{0}$ is the quadratic part of $\mathcal{L}_{\bullet}^{\text {no }}$, hence including the gauge fixing: $\mathcal{L}_{0}=-\left(F^{a}\right)^{2} / 4-\left(\partial A^{a}\right)^{2} /(2 \alpha$. $)$. The FaddeevPopov determinant now depends on the gauge field and is 
thus subject to functional integrations. But for convenience we may split off its bare factor. The partition function, still waiting for its even-odd decomposition, so far reads

$$
Z .=\frac{1}{Z_{B}^{\cdot}} \operatorname{det}^{\prime}\left(\beta^{2} \partial^{2} \delta^{a b}\right) \mathcal{N} \int \mathcal{D} A_{\mu}^{a} e^{-S_{\cdot}^{\mathrm{no}}-S_{\mathrm{FP}} .}
$$

The two factors in Eq. (68), which obviously stand for the FP determinant $\operatorname{det}^{\prime}\left(\beta^{2} \partial D\right)$, derive through

$$
\begin{aligned}
\operatorname{det}^{\prime}\left(\beta^{2} \partial D\right)= & \operatorname{det}^{\prime}\left(\beta^{2} \partial^{2} \delta^{a b}\right) \\
& \times \operatorname{det}^{\prime}\left(\left[\partial^{2} \delta^{a b}-\partial^{\mu} g f^{a b c} A_{\mu}^{c}\right] \frac{1}{\partial^{2} \delta^{a b}}\right) \\
& \equiv \operatorname{det}^{\prime}\left(\beta^{2} \partial^{2} \delta^{a b}\right) \operatorname{det}^{\prime}(1+\mathcal{W}) \\
& \equiv \operatorname{det}^{\prime}\left(\beta^{2} \partial^{2} \delta^{a b}\right) e^{-S_{\mathrm{FP}}}
\end{aligned}
$$

where by $\mathcal{W}$ the part odd in the gauge field is prepared:

$$
\mathcal{W}=-g f^{a b c} \partial^{\mu} A_{\mu}^{c} \frac{1}{\partial^{2}} .
$$

The first $\partial^{\mu}$ acts on $A_{\mu}^{c}$ and all functions that follow. We read off from Eq. (69) that

$$
\begin{aligned}
S_{\mathrm{FP}} & =-\ln \left[\operatorname{det}^{\prime}(1+\mathcal{W})\right]=-\operatorname{Tr}^{\prime} \ln (1+\mathcal{W}) \\
& =-\frac{1}{2} \operatorname{Tr}^{\prime} \ln \left(1-\mathcal{W}^{2}\right)-\frac{1}{2} \operatorname{Tr}^{\prime} \ln \left(\frac{1+\mathcal{W}}{1-\mathcal{W}}\right) .
\end{aligned}
$$

In the second line, clearly, the even-odd decomposition is achieved. But the second equality in Eq. (71) (first line) is delicate, because all eigenvalues of $1+\mathcal{W}$ have to be positive, but are not. While this point needs care in exactly solvable models [38], here we may be content with a crude argument. For the intended comparison with perturbation theory, the above logarithms are expanded anyway. Hence Eq. (71) is merely a formal compact notation for series to be generated [39].

We are ready to form the non-Abelian "even" action $S$.. through $S_{.}^{\text {no }}+S_{\mathrm{FP}} \rightarrow S . .=\mathcal{E}-\ln [\cosh (\mathcal{O})]$ with $\mathcal{E}, \mathcal{O}$ given by

$$
\begin{gathered}
\mathcal{E}=-\int^{\beta}\left(\mathcal{L}_{0}+\mathcal{L}_{2}\right)-\frac{1}{2} \operatorname{Tr}^{\prime} \ln \left(1-\mathcal{W}^{2}\right), \\
\mathcal{O}=-\int^{\beta} \mathcal{L}_{1}-\frac{1}{2} \operatorname{Tr}^{\prime} \ln \left(\frac{1+\mathcal{W}}{1-\mathcal{W}}\right) .
\end{gathered}
$$

The trial theory has remained unchanged. It is that of Sec. III. The trial partition function is given by Eq. (68) without the bullets, at $S_{\mathrm{FP}}=0$ and with $S^{\text {no }}=-\int^{\beta} \mathcal{L}^{\text {no }}$. The free energy $F$ is Eq. (30). Thus, the "even" functional (23) of the gluon system (taken at $\alpha=\alpha$.) reads

$$
\begin{aligned}
\mathcal{V}= & F+T\left\langle\int \frac{1}{2} A^{a}\left(M A^{a}\right)-\int^{\beta} \mathcal{L}_{2}-\frac{1}{2} \operatorname{Tr}^{\prime} \ln \left(1-\mathcal{W}^{2}\right)\right. \\
& \left.-\ln \left[\cosh \left(\int^{\beta} \mathcal{L}_{1}+\frac{1}{2} \operatorname{Tr}^{\prime} \ln \left[\frac{1+\mathcal{W}}{1-\mathcal{W}}\right]\right)\right]\right\rangle .
\end{aligned}
$$

The first two terms form the bare part $\mathcal{V}_{0}$ and are familar from Secs. III and IV:

$$
\mathcal{V}_{0}=n V T^{4}\left(-2 I_{t}-L_{t}-I_{l}-\frac{1}{2} L_{l}+I_{0}\right) .
$$

As in Sec. IV, we expand the logarithms up to $\mathcal{W}^{2}$ to reach a reasonable simple "truncated version." Since $\operatorname{Tr}^{\prime} \mathcal{W}=0$, no such term arises from the last logarithm. Thus,

$$
\mathcal{V}_{\text {trunc }}=\mathcal{V}_{0}+\mathcal{V}_{\text {int }}, \quad \mathcal{V}_{\text {int }}=\mathcal{V}_{A A A A}+\mathcal{V}_{\mathrm{FP}}+\mathcal{V}_{\text {square }}
$$

with

$$
\mathcal{V}_{\mathrm{FP}}=\frac{T}{2}\left\langle\operatorname{Tr}^{\prime} \mathcal{W}^{2}\right\rangle, \quad \mathcal{V}_{\text {square }}=-\frac{T}{2}\left\langle\left(\int^{\beta} \mathcal{L}_{1}\right)^{2}\right\rangle .
$$

The contribution $\mathcal{V}_{A A A A}$ is given by Eq. (28). It agrees with the perturbative free energy contribution from the tadpole diagram (both lines gluons): $\mathcal{V}_{A A A A}=F^{\infty}$. Compared to Sec. III, there are two additional terms in Eq. (76): the last two. By analogy with Sec. IV we expect that they equal the two other diagrams at second order, which were missing in Sec. III. Indeed, taking the trace of $\mathcal{W}^{2}$ with states $(\beta V)^{-1 / 2} e^{-i P x}$, using $f^{a b c} f^{a b c}=N n$ and through Wick decomposition, we obtain

$$
\begin{aligned}
\mathcal{V}_{\mathrm{FP}}= & n V \frac{g^{2} N}{2} \sum_{Q} \sum_{P} G_{\mu \nu}(Q) \frac{P^{\mu}(P-Q)^{\nu}}{P^{2}(Q-P)^{2}}=F^{\doteqdot} \\
\mathcal{V}_{\text {square }}= & n V \frac{g^{2} N}{2} \sum_{Q} \sum_{P}\left[(Q+P)^{\lambda} G_{\lambda \nu}(Q-P) G^{\nu \rho}(Q)\right. \\
& \left.-G_{\lambda \tau}(Q) G^{\lambda \tau}(Q-P) Q^{\rho}\right] G_{\rho \mu}(P)(2 Q-P)^{\mu} \\
= & F_{\bullet}^{\ominus}
\end{aligned}
$$

where, in Eq. (78), the symbol $\div$ (with two out of many dots) stands for the ghost loop with an inner gluon line.

Quite different from scalar ED, the gauge-fixing dependence does not cancel in a manner independent of variational parameters. Splitting the Green's function as $G=\chi$ $+\alpha \mathrm{D} \Delta_{0}$, we see that $\alpha$ occurs up to the third power. The term $\alpha^{3}$ is contained in $\mathcal{V}_{\text {square }}$ only, and its prefactor vanishes. Collecting $\alpha^{2}$ and $\alpha$ terms one obtains

$$
\begin{aligned}
\mathcal{V}^{\left(\alpha^{2}\right)}= & -n V \frac{g^{2} N}{4} \alpha^{2} \sum_{Q} \sum_{P} \frac{Q^{4}}{P^{4}(Q-P)^{4}} P^{\mu} \chi_{\mu \nu}(Q) P^{\nu} \\
& -(\text { the same at zero mass }), \\
\mathcal{V}^{(\alpha)}= & n V \frac{g^{2} N}{2} \alpha \sum_{Q} \sum_{P} \frac{1}{(Q-P)^{4}}\left[\chi_{\mu}^{\mu}(Q)(Q-P)^{2}\right. \\
& \left.-P^{\mu} \chi_{\mu \nu}(Q) P^{\nu}+Q^{2}\left(P^{2}-Q^{2}\right) \chi_{\mu \nu}(Q) \chi^{\mu \nu}(P)\right] \\
& -(\text { the same at zero mass }) .
\end{aligned}
$$

The fact that Eqs. (80) and (81) vanish at zero mass reflects gauge invariance of thermodynamic perturbation theory at order $g^{2}$. For the next step, namely analyzing $\mathcal{V}_{\text {trunc }}$ at order $g^{3}$, we need more: Eqs. (80) and (81) must remain below $g^{3}$. This is the case, as one may check, e.g., by power count- 
ing. Remember that perturbatively a $g^{3}$ only arises by dressing the $g^{2}$ diagrams, whereby gauge invariance persists.

The strategy of further evaluation is now that of Sec. IV, as detailed above Eq. (53). Since they are of higher order, we temporarily omit the two $\alpha$-dependent terms (80) and (81). In $\mathcal{V}_{\text {int }}$ this amounts to the replacement $G \rightarrow \chi=\mathrm{A} \Delta_{t}$ $+\mathrm{B} \Delta_{l}$. Then the terms $\left(v_{1}\right)$ linear in $\chi-\chi_{0}$ are isolated, and terms of higher order-others than in Sec. IV-move to $v_{2}$. But evaluation of $v_{1}$ runs through the steps in Sec. IV B and, surprisingly, ends up with the same result as in Sec. IV, namely Eq. (57) at $m_{s}=0$. Just to show the prefactors

$$
\begin{gathered}
\mathcal{V}_{\mathrm{int}}=n V g^{2} N\left(v_{0}+v_{1}+v_{2}\right), \quad v_{0}=\frac{T^{4}}{144}, \\
v_{1}=\frac{T^{4}}{g^{2} N \lambda_{t}^{2}} L_{t}+\frac{T^{4}}{2 g^{2} N \lambda_{l}^{2}} L_{l}-\frac{T^{4}}{6} J_{0} .
\end{gathered}
$$

The complete functional up to order $g^{3}$ [add Eq. (82) to Eq. (75)] does not depend on $\lambda_{t}$ (gutter form) and reads

$$
\mathcal{V}^{\text {to } g^{3}}=\text { const }+n V T^{4} \frac{1}{2} \mathcal{U}_{l}
$$

with the function $\mathcal{U}_{l}$ defined as $\mathcal{U}_{t}$ in Eq. (64) by changing the index. Minimization gives $\lambda_{l}=1$, as desired. For the height of the minimum to order $g^{3}$ we obtain

$$
\mathcal{V}^{\min }=n V T^{4}\left[-\frac{\pi^{2}}{45}+\frac{g^{2} N}{144}-\frac{1}{12 \pi}\left(g \sqrt{\frac{N}{\sqrt{3}}}\right)^{3}\right] .
$$

This is Eq. (8.47) in [21]. At $N=1$, the correlation energy $\left(g^{3}\right.$ term) agrees with the photonic one in scalar ED, see Eq. (63).

As in the Abelian case (Sec. IV D) the functional is expected to become convex with respect to $\lambda_{t}$ by including $g^{4}$ terms. However, at this point we run into non-Abelian difficulties. There are four terms to be included. The first one is $\mathcal{U}_{t}$ [replace $\mathcal{U}_{l}$ in Eq. (83) by $\mathcal{U}_{l}+2 \mathcal{U}_{t}$ ], which has a minimum at $\lambda_{t}=1$. The second term arises from $v_{2}$ in Eq. (82), a rather lengthy expression (seven lines say) and so far not evaluated. The third and fourth terms are the $\alpha$-dependent pieces (80) and (81) and cause the trouble. They should be (but are not) either constant, or minimal at $\lambda_{t}=1$, too, or of lower order in magnitude. Consider, e.g., the $\Delta_{t 0}$ part of the $\alpha^{2}$ term (80). If evaluated "soft" it vanishes (in the manner noted at the end of Sec. IV). At first glance, as no UV cutoff is needed, one might conclude that $\mathcal{V}_{t}^{\left(\alpha^{2}\right)}=0$ at all. However, it appears that there is still a hard contribution, which in turn needs no IR cutoff. Because this is perhaps somewhat unusual, let us state the result

$$
\mathcal{V}_{t}^{\left(\alpha^{2}\right)}=-n V T^{4} \frac{\alpha^{2}}{24} \frac{g^{4} N^{2} \lambda_{t}^{2}}{48 \pi^{4}} \mathfrak{I}
$$

with

$$
\begin{aligned}
\mathfrak{I}= & \int_{0}^{\infty} d x \frac{x}{e^{x}-1} \int_{0}^{\infty} d t \frac{1}{e^{(1 / 2) x t}-1}\left(\frac{t}{t^{2}-1}+\frac{4 t}{\left(t^{2}-1\right)^{2}}\right. \\
& \left.+\frac{1}{(t+1)^{3}} \ln (t+2)+\frac{1}{(t-1)^{3}} \ln |t-2|\right) .
\end{aligned}
$$

The derivation used Eq. (C2). To check the above statement of vanishing soft part, one may write $2 /(x t)$ for the second Bose function. Then the integral over $t$ gives zero, as required. But as it stands, $\mathfrak{I}$ is some nonzero mathematical constant $(\mathfrak{I} \approx-1.04)$.

The above remaining $\alpha$ dependence, which prevents us from solving the gutter problem in the non-Abelian case, is the "minor detail" noted in pt. (8) of the Introduction. There must be a resolution to this puzzle within the truncated version (76), because the terms beyond, depending on $\lambda$, are of order $g^{5}$. As the term (85) contains two Bose functions, the way out has probably nothing to do with renormalizations. The only possibility we are able to invent is the fact that at higher orders there is also a $C$ term [see Eq. (5)] in the propagator, which is missing in Eq. (4) and is specific to non-Abelian theory. Furthermore, this term has a factor $\alpha$ in front of it; see, e.g., Sec. 3 of [40]. Let such speculations be beyond the scope of the present paper.

\section{STATIC PROPERTIES}

So far, while testing the "even version" in the $\lambda$ case, we were thinking in terms of real excitations in the plasma (scalar and gluon), whose spectra are hidden in the polarization functions. Here we recall the other well-tractable case within the infinity of Abelian gauge invariant mass terms. Before all, turning to the $m$ case comes with a change in philosophy. We now ask for the best constant-mass terms (longitudinal and transverse) in the trial Lagrangian. To leading order (otherwise see e.g., [34]), static propagators have the form $\left(-q^{2}-m_{\text {screen }}^{2}\right)^{-1}$. But the trial propagators read $\left(Q_{0}^{2}\right.$ $\left.-q^{2}-m_{t, l}^{2}\right)^{-1}$. Nevertheless, it may well happen (remember the " $Q_{0}=0$ method" of Sec. IV B) that they lose memory to their dynamical element $Q_{0}^{2}$ automatically.

For Yang-Mills fields, the analysis runs through the steps of Sec. V up to Eq. (82). No gauge-fixing dependence occurs up to the order $g^{3}$ to be considered here. The bare part $\mathcal{V}_{0}$ is given by Eq. (75), now with the $m$-case integrals (36) and (37) to be inserted. The crucial line where the $m$ case starts to make differences reads

$$
v_{1}=2 T^{4} J_{0}\left(J_{t}-J_{0}\right)-2 \sum_{Q} \Delta_{l t}(Q) \Pi_{l}(Q) .
$$

Within the present accuracy, the above sum may be reduced to its $Q_{0}=0$ term. But note the difference to the $\lambda$ case. Once the transverse propagator is supplied with a nonzero magnetic mass by hand, this variational parameter survives in the result:

$$
\sum_{Q} \Delta_{l t} \Pi_{l}=\frac{T^{4} g^{2} N}{12 \pi}\left(\beta m_{l}-\beta m_{t}\right) .
$$

The same happens in the $J_{t}$ sum, see Eq. (37). But the combination of these details in Eq. (86) yields $v_{1}=-T^{3} m_{l} /$ 
$(24 \pi)$. The linear (not the cubic, see below) dependence on $m_{t}$ has gone, this time by cancellation-a wanted detail, as we see next. Including the bare part $\mathcal{V}_{0}$ the functional reads

$$
\begin{aligned}
\mathcal{V}_{m}^{\text {to }} \stackrel{g}{\text { case }}^{3}= & n V T^{4}\left(-\frac{\pi^{2}}{45}+\frac{g^{2} N}{144}+\frac{1}{24 \pi}\left[\left(\beta m_{l}\right)^{3}-g^{2} N \beta m_{l}\right]\right. \\
& \left.+\frac{1}{12 \pi}\left(\beta m_{t}\right)^{3}\right)
\end{aligned}
$$

The longitudinal part clearly becomes minimal at $m_{l}=g \sqrt{N} T / \sqrt{3}$, which is the well known Debye screening mass at leading order. There is a transverse part in Eq. (88), hence no gutter problem. As $m_{t}$ is restricted to the positive half-axis, the minimum is reached at $m_{t}=0$, which is the magnetic mass at the order studied, indeed.

In spite of the above correct answers on static properties, there remain delicate questions. Remember that the (squared) Debye mass $3 \mathrm{~m}^{2}$ already entered the dynamical calculation at Eq. (58). It appears that, within the order $g^{3}$, the variational functional cannot really discriminate between statics and dynamics. In fact, the minimum value of the functional (88) agrees with Eq. (84), i.e., with the exact one to order $g^{3}$. Thus, two equally low minima are found over the space of mass terms. However they are joined, namely through a subspace of all functions $\Pi_{l}$ that have the value $3 \mathrm{~m}^{2}$ at zero frequency, and $\Pi_{t}$ vanishing there. Nevertheless, in the $\lambda$ case the appearence of constant masses is a technical byproduct, while in the present static case it answers the posed question. Let us add conjectures on the behavior in higher orders. The safe ground is on the dynamical side. Supplying the variational functional with anything good, then it might answer with self-energies comparably good. For static properties, on the other hand, one needs more, namely some philosophy of why the trial propagators get rid of its dynamical part $Q_{0}^{2}$ by only forcing the mass to be constant. Remember also that, starting from the real-excitation spectrum in the $\omega-q$ plane, the static limit $(\omega=0)$ is only reached through a range with imaginary wave vector [36] on mass-shell lines. Perhaps the variational procedure prepares at least the first nonvanishing term of each screening mass.

At the supersoft scale, the magnetic mass (see [33,41] for more recent work) most probably comes with some numerical factor times $g^{2} T$ [42]. Then, as a rough speculation, the last term in

$$
\mathcal{V}=n V T^{4}\left(\operatorname{const}+\frac{1}{12 \pi}\left(\beta m_{t}\right)^{3}-\text { const } g^{4} \beta m_{t}\right)
$$

would be in search. Note that such a term, if any and if no others, would arise in one step over the present truncation of the functional. For possible danger with this step see the last point in the following list of open questions.

For completeness, we add the $m$-case result for scalar electrodynamics. It simply agrees with Eq. (88) at $N=n=1$, except for the constant terms and an additional term due to the scalars, which may be both read off from Eq. (61). Let us end up with the question which way the magnetic sectors of Abelian [43] and non-Abelian theories might become different in a variational treatment.

\section{OPEN QUESTIONS}

In the preceding sections, the application of the variational calculus to pure gauge theory was far from being a straightforward procedure. Several problems were eluded and questions not answered, because we could not. Let us recall these questions and just list them here.

(1) The Hamiltonian formulation to both, the GibbsBogoljubov or Feynman-Jensen varational principle [see text below Eq. (17)], was given up in Sec. II because we were unable to construct the Hamiltonian $H$ of the trial theory. This construction is a challenging task. See the text below Eq. (16).

(2) Knowing the Hamiltonians of both, trial and studied theory, one could construct the common physical Hilbert space. By forming the Becchi-Rouet-Stora-Tyutin (BRST) charge and projecting out physical states from the outset, this would be the natural approach to the Gibbs-Bogoljubov version $[6,8,15]$.

(3) The functional $V$ in both versions, Gibbs-Bogoljubov and Feynman-Jensen, has the total minimum value in common (namely the exact free energy). However, the trial spaces are different. Hence, a given trial theory which does not cover this minimum could lead to quite different approximations. Since presumedly, this is not true, a proof of the full equivalence of the two principles is desirable. Note that such a proof would circumvent our Hamiltonian problem of the above point (1). Moreover, the interpretation of the trial space as one of nonequilibrium statistical operators would be preserved.

(4) We have not made an effort to introduce, by Legendre transformation, the 1PI-generating functional $\Gamma$, although there is a variational principle even to $\Gamma[44,29]$.

(5) Renormalization [6], not yet needed in this paper, is probably inevitable already when the method should reproduce the next-to-leading-order perturbative results, such as, e.g., the lowering "by glue", of the longitudinal plasma frequency [for scalar ED this is the term $-0.37 e$ in Eq. (5.5) of [19]].

(6) From Secs. II B to II C we turned to the "even version" immediately. But perhaps there is something in between that we have not found, namely a feasible modified trial theory not running into the pitfall of Sec. III.

(7) Only a very poor subspace of polarization functions was considered by simply varying prefactors $\lambda_{t, l}$ in front of the true functions $\Pi_{t}, \Pi_{l}$, already known perturbatively. An honest "even version"-variational treatment might instead vary unknown functions $\Pi_{t, l}(Q)$. To make sense, this generalization probably needs $g^{4}$ terms in the functional $\mathcal{V}$.

(8) For the "minor detail" of reminescent $\alpha$ dependence when solving the gutter problem in the non-Abelian case, see the comments at the end of Sec. V.

(9) The most troubling step in Secs. IV and V was the expansion of the $\ln [\cosh ()]$ term in the variational functional. So, the question is whether this expansion can be avoided some way.

(10) With regard to the observed gauge-fixing independence, it could turn out that a later truncation of the series makes less sense than reading $\ln [\cosh (x)] \approx \frac{1}{2} x^{2}$ as some good approximation. 


\section{CONCLUSIONS}

A Feynman-Jensen type thermal variational principle is constructed such that an Abelian free trial theory works well in both cases, scalar electrodynamics and pure Yang-Mills theory. To this end their actions are to be rewritten such that only even powers in the gauge field appear. This way, the perturbatively known leading-order self-energies of photons, scalars, and gluons, respectively, are reproduced (apart from a minor open question to the non-Abelian case) by variation of their prefactors. The subspace of constant masses covers the inverse Debye screening length. There is a large asymmetry of the functional with respect to the (photonic/gluonic) transverse sector, as it does not (yet) depend on the corresponding parameter at order $g^{3}$.

The delicate problem of handling two different covariant gauge-fixing parameters (one of the original and one of the trial theory) has a simple resolution: they become equal by minimization. Hence, the observed gauge independence refers to the remaining gauge-fixing parameter common to both theories.

The new variational functional contains a term $\ln [\cosh (A A A)]$ and hence involves arbitrarily high even powers of the gauge fields $A$. In the non-Abelian case (and within covariant gauges) such powers occur already in the unmodified Feynman-Jensen principle due to the FaddeevPopov determinant depending on $A$. Unfortunately, for evaluation and minimization we had to expand the ln-cosh function. But a true nonperturbative scheme should never refer to $g$ powers at all. So, the present success is still below the potential nonperturbative possibilities of the variational approach.

\section{ACKNOWLEDGMENTS}

We are very indebted to Martin Reuter, who made us aware of the Feynman formulation and correctly localized the origin of our initial problems in the FP determinant. We are also grateful to specific hints and valuable discussions with Norbert Dragon, Fritjof Flechsig, Edmond Iancu, Olaf Lechtenfeld, Anton Rebhan, and Andreas Wipf.

\section{APPENDIX A}

Here the functional integral measure of the trial partition function $Z$ of scalar ED is made explicit. $Z$ is Eq. (43) without the dots there. The normalization factor $\mathcal{N}$ is fixed by requiring that, in the massless limit, the partition function $Z$ must turn into two times that of blackbody radiation, one of the photons and one of the scalars. On the more ambitious task of a true derivation see the comments at the end of this Appendix.

We start by splitting $Z$ into four factors, $Z=Z_{\alpha} Z_{\text {det }} Z_{A} Z_{s}$ with a piece of $\mathcal{N}$ contained in each. But notice the redundance of such a factor in front of an unspecified $\int \mathcal{D} \ldots$, hence, e.g., $Z_{\alpha}=1 / Z_{B}$ suffices. The simplest part is $Z_{s}=$ " $\int \mathcal{D}\left\{\phi^{*} \phi\right\}$ ' $e^{-S_{s}}$ with

$$
\begin{aligned}
S_{s} & =\int^{\beta}\left(m_{s}^{2} \phi^{*} \phi+\phi^{*} \partial^{2} \phi\right)=\sum_{P}\left(m_{s}^{2}-P^{2}\right) \phi(P)^{*} \phi(P) \\
& =\sum_{\mathbf{p}, n} \frac{m_{s}^{2}-P^{2}}{\beta V} \phi^{*} \phi .
\end{aligned}
$$

At each of the countable infinite discrete positions $\mathbf{p}, n$ there are, as $\phi$ is complex, two independent integrations. Equation (A1) refers to our convention $\phi(x)=\sum e^{-i P x} \phi(P)$ but we may turn to that of Kapusta [21] by $\phi(P)$ $=\sqrt{\beta V}(a+i b) / \sqrt{2}$ (with indices $\mathbf{p}, n$ on $a, b$ suppressed). We now guess the functional integral measure and justify by evaluation:

$$
\begin{aligned}
Z_{s} & =\mathcal{N}_{0}^{2} \prod_{\mathbf{p}, n} \frac{1}{2 \pi \beta^{2}} \int d a d b \exp \left[-\frac{1}{2}\left(m_{s}^{2}-P^{2}\right)\left(a^{2}+b^{2}\right)\right] \\
& =\mathcal{N}_{0}^{2} \prod\left(-T^{2} \Delta_{s}\right),
\end{aligned}
$$

where $\Pi \equiv \Pi_{\mathbf{p}, n}$ and

$$
\mathcal{N}_{0}=\prod_{\mathbf{p}} \prod_{n}^{\prime} 2 \pi n
$$

with the prime excluding $n=0$. Remember that $\Delta_{s}^{-1}$ $=P^{2}-m_{s}^{2}=-(2 \pi n T)^{2}-\mathbf{p}^{2}-m_{s}^{2}<0$. Of course, each factor in $\mathcal{N}_{0}$ has to be attached to the corresponding one in the $n$ product in Eq. (A2), and the product over $n$ has to be performed first (other constructions may be possible).

The infinite product (A2) can be performed. To this end we collect four (known) formulas of general use. By contour integration,

$$
T \sum_{n} \frac{1}{P_{0}^{2}-x^{2}}=-\frac{1}{x}\left[\frac{1}{2}+n(x)\right]
$$

with $n(x) \equiv 1 /\left(e^{\beta x}-1\right)$ the Bose function. Equation (A4) is Eq. (2.38) of [21]. Multiply Eq. (A4) with $2 x$ and integrate over $x$ from $c$ to $y$ :

$$
T \sum_{n} \ln \left(\frac{y^{2}-P_{0}^{2}}{c^{2}-P_{0}^{2}}\right)=y-c+2 T \ln \left(\frac{1-e^{-\beta y}}{1-e^{-\beta c}}\right) .
$$

Multiply Eq. (A5) with $\beta$, set $\beta y=\omega$, and perform the limit $\beta c \rightarrow 0$ :

$$
\sum_{n=1}^{\infty} \ln \left(1+\frac{\omega^{2}}{(2 \pi n)^{2}}\right)=\ln \left(\frac{\sinh (\omega / 2)}{\omega / 2}\right) .
$$

Exponentiating Eq. (A6) and extending to all $n$, one arrives at the fourth formula

$$
\prod_{n}^{\prime} \frac{(2 \pi n)^{2}}{\omega^{2}+(2 \pi n)^{2}}=\frac{\omega^{2} e^{-\omega}}{\left(1-e^{-\omega}\right)^{2}},
$$

which is Eq. (89.5.16) in [45] and Eq. (2.269) in [12]. Check Eq. (A7) at $\omega \rightarrow 0$. Using Eq. (A7) for Eq. (A2) we obtain 


$$
\begin{gathered}
Z_{s}=\mathcal{N}_{0}^{2} \prod\left(-T^{2} \Delta_{s}\right)=\prod_{\mathbf{p}} \frac{e^{-\beta \sqrt{m_{s}^{2}+p^{2}}}}{\left(1-e^{\left.-\beta \sqrt{m_{s}^{2}+p^{2}}\right)^{2}}\right.}, \quad \text { i.e. } \\
F_{s}=-T \ln \left(Z_{s}\right)=2 \sum_{\mathbf{p}}\left[\frac{1}{2} \sqrt{m_{s}^{2}+p^{2}}\right. \\
+T \ln \left(1-e^{\left.-\beta \sqrt{m_{s}^{2}+p^{2}}\right)}\right]
\end{gathered}
$$

which is, at zero mass, the desired result of twice a half blackbody radiation. The guessing was good. Aside, one could include the zero-point energies by the redefinition $\mathcal{N}_{0} \rightarrow \Pi_{\mathbf{p}} e^{\beta p / 2} \Pi_{n}{ }^{\prime} 2 \pi n$.

We turn to the factor $Z_{\text {det }}$ with again a trial-and-error prefactor:

$$
\begin{aligned}
Z_{\mathrm{det}} & =\mathcal{N}_{0}^{-2} \operatorname{det}^{\prime}\left(\beta^{2} \partial^{2}\right)=\left[\mathcal{N}_{0}^{2} \Pi^{\prime}\left(-T^{2} \Delta_{0}\right)\right]^{-1} \\
& =\left[\prod_{\mathbf{p}}^{\prime}(\beta p)^{2}\right]\left[\prod_{\mathbf{p}} \prod_{n}^{\prime} \frac{(\beta p)^{2}+(2 \pi n)^{2}}{(2 \pi n)^{2}}\right],
\end{aligned}
$$

where in the blank $\Pi^{\prime}$ and on the determinant the prime excludes only the one position $n=\mathbf{p}=0$. As the determinant is the product of the eigenvalues $-\beta^{2} P^{2}$, naively, $P=0$ must be excluded to make sense. However, if this is required to result from a derivation, one might go back to the unity insertion in the Faddeev-Popov procedure:

$$
1=\Delta \cdot \mathcal{N}_{0}^{2}\left[\prod^{\prime} T^{2} \int d a_{\mathbf{p}, n}\right]\left[\prod^{\prime} \delta\left(-P^{2} a_{\mathbf{p}, n}\right)\right]
$$

Originally the $\delta$ argument was $\partial^{2} \Lambda$ (with $\delta A_{\mu}=-\partial_{\mu} \Lambda$ the gauge variation). Since there is no constant term in $\Lambda$, there is no $a_{\mathbf{0}, 0}$ integration in Eq. (A12) and no zero $P$ in Eq. (A10), Q.E.D. Equation (A12) directly leads to $\Delta=Z_{\text {det }}$. Using Eq. (A7) for Eq. (A11) we have

$$
F_{\text {det }}=-T \ln \left(Z_{\mathrm{det}}\right)=-2 \sum_{\mathbf{p}}{ }^{\prime}\left[\frac{1}{2} p+T \ln \left(1-e^{-\beta p}\right)\right] \text {. }
$$

Clearly, with the above measure, the determinant term subtracts twice a half blackbody radiation. In passing, the prime in Eq. (A13), while still being necessary there, becomes irrelevant in the continuum limit.

With an argument quite similar to that below Eq. (A12), there is also a prime in the measure of $\int \mathcal{D} B$. This integration runs over a $\delta(\partial A-B)$. But $\partial A$ cannot be constant, since otherwise $A$ would be linear in spacetime and lie outside our space of Fourier-transformable fields. So, $P=0$ may be excluded:

$$
\begin{aligned}
Z_{B} & =“ \int \mathcal{D} B{ }^{\prime \prime} \exp \left(-\frac{1}{2 \alpha} \sum_{P}^{\prime} B(P)^{*} B(P)\right) \\
& =\prod \frac{1}{2 \pi} \int d a d b \exp \left(-\frac{1}{2 \alpha}\left(a^{2}+b^{2}\right)\right)=\prod^{\prime} \sqrt{\alpha} .
\end{aligned}
$$

As $B(x)$ is a real field and $B(-P)^{*}=B(P)$, $B(P)=\sqrt{\beta V}(a+i b) / \sqrt{2}$, the two integrations are placed on half of the $P$ space, the right say (let right and left exclude the origin). The prefactor was chosen here to reach the simple result $Z_{\alpha}=1 / Z_{B}=\Pi^{\prime} \sqrt{1 / \alpha}$. It must wait to make sense in combination with $Z_{A}$.

The photonic part of the trial action includes the mass terms $M_{t, l}$ :

$$
\begin{aligned}
S_{A}= & \frac{1}{2} \sum_{P}\left(P^{2}-M_{t}\right) A_{\mu}^{-} \mathrm{A}^{\mu \nu} A_{\nu}+\frac{1}{2} \sum_{P}\left(P^{2}-M_{l}\right) A_{\mu}^{-} \mathrm{B}^{\mu \nu} A_{\nu} \\
& +\frac{1}{2 \alpha} \sum_{P} P^{2} A_{\mu}^{-} \mathrm{D}^{\mu \nu} A_{\nu} \equiv S_{A}^{t}+S_{A}^{l}
\end{aligned}
$$

with $A_{\mu}^{-}=A_{\mu}(-P)$ and the $\mathrm{D}$ term being part of $S_{A}^{l}$. The corresponding further splitting $Z_{A}=Z_{A}^{t} Z_{A}^{l}$ is allowed because the transverse components (those in $\mathbf{e}_{1,2}$ direction, $\mathbf{e}_{1,2} \perp \mathbf{p}$, $\left.\mathbf{e}_{1} \perp \mathbf{e}_{2}\right)$ in the expansion

$$
\begin{gathered}
A^{\mu}(P)=u_{1} E_{1}^{\mu}+u_{2} E_{2}^{\mu}+v T^{\mu}+i w U^{\mu} \text { with } T^{\mu}=\left(0, \frac{\mathbf{p}}{p}\right), \\
E_{1,2}^{\mu}=\left(0, \mathbf{e}_{1,2}\right),
\end{gathered}
$$

drop out in $S_{A}^{l}$ and are the only parts surviving under the $\mathrm{A}$ operation: $\mathrm{A}^{\mu \nu} E_{1,2 \nu}=E_{1,2}^{\mu}$. As the first three terms of Eq. (A16) as well as $w U^{\mu}$ are Fourier transforms of real fields, half $P$ spaces are related by $u_{j}(-P) E_{j}^{\mu}(-P)$ $=u_{j}(P)^{*} E_{j}^{\mu}(P), v(-P)=-v(P)^{*}$ and $w(-P)=w(P)^{*}$. Hence, the integrations in $Z_{A}^{t}$ to start with, are of the realfield type (A14), except that there are now two integrations at the origin $n=\mathbf{p}=0$ and four at each place in the right half. Two of the latter may be attached with the left half. Then, choosing the same functional integral measure as for $Z_{s}$, we arrive at precisely Eq. (A2) with the role of $m_{s}^{2}$ taken over by $M_{t}(P)$ :

$$
Z_{A}^{t}=\mathcal{N}_{0}^{2} \prod\left(-T^{2} \Delta_{t}\right)
$$

The longitudinal part of the action is first rewritten as

$$
S_{A}^{l}=\frac{1}{2} \frac{1}{\beta V} \sum_{\mathbf{p}, n}^{\text {all }}\left(\left[M_{l}-P^{2}\right]|\xi|^{2}-P^{2} \frac{1}{\alpha}|\eta|^{2}\right)
$$

with

$$
\xi=p w+i P_{0} v, \quad \eta=-i P_{0} w+p v
$$


Next we observe that $\xi(-P)=\xi(P)^{*}, \eta(-P)=-\eta(P)^{*}$, and mark the origin and the right half $P$ space to count independent integrations (two over $\xi$ at the origin and four in the right). Finally, by changing the variables from $v, w$ to $\xi, \eta$ (with unit Jacobian determinants), and with the now familiar functional integral measure, one arrives at

$$
Z_{A}^{l}=\mathcal{N}_{0} \prod \sqrt{-T^{2} \Delta_{l}} \mathcal{N}_{0} \prod^{\prime} \sqrt{-T^{2} \alpha \Delta_{0}} .
$$

Note that most of the above "trivialities" were due to carefully counting all positions in $P$ space, i.e., to place the primes right.

We are ready to constitute the scalar ED partition function from the above several factors:

$$
\begin{aligned}
Z= & \frac{1}{\Pi^{\prime} \sqrt{\alpha}} \frac{1}{\mathcal{N}_{0}^{2} \Pi^{\prime}\left(-T^{2} \Delta_{0}\right)} \mathcal{N}_{0}^{2} \Pi\left(-T^{2} \Delta_{t}\right) \\
& \times \sqrt{\mathcal{N}_{0}^{2} \Pi\left(-T^{2} \Delta_{l}\right)} \\
& \times \sqrt{\left[\Pi^{\prime} \alpha\right] \mathcal{N}_{0}^{2} \Pi^{\prime}\left(-T^{2} \Delta_{0}\right)} \mathcal{N}_{0}^{2} \Pi\left(-T^{2} \Delta_{s}\right) .
\end{aligned}
$$

Obviously, the gauge-fixing parameter $\alpha$ cancels. Now, counting halves of blackbody radiation amounts to $-2+2+1+1+2=4$ as required.

A true derivation of the above must not anticipate the known zero-mass results. With [26] as a guideline, such derivation should be possible even inside covariant gauges, i.e., without a recourse to physical gauges. There is one problem in taking the right starting point [maybe with a factor $\mathcal{N}_{0}^{4}$ in front of the classical partition function for the four (of six) degrees of freedom to be quantized], and in the volume factor (to be split off) the other.

\section{APPENDIX B}

Here the two sums $L_{t}$ and $L_{t}$ are evaluated, in the $\lambda$ case and with regard to contributions not accessible by a naive $Q_{0}=0$ method. The details are required for Secs. IV B and IV D. We start from the definition (26) and work with the spectral representation

$$
\frac{1}{P^{2}-\lambda^{2} \Pi_{t, l}(P)}=\int d x x \frac{\rho_{t, l}^{(\lambda)}(x, p)}{P_{0}^{2}-x^{2}},
$$

of trial propagators. The above spectral densities are related to ordinary ones, denoted by $\rho_{t, l}\left(x, p ; m^{2}\right)$, by

$$
\rho_{t, l}^{(\lambda)}(x, p)=\rho_{t, l}\left(x, p ; \lambda^{2} m^{2}\right) .
$$

Hence all sum rules (C6) and (C7) remain valid for $\rho^{(\lambda)}$ if $m^{2}$ is replaced by $\lambda^{2} m^{2}$ to the right. Using Eq. (B1) and the sum rule $1=\int d x x \rho^{(\lambda)}(x, p)$ the $L$ sums read

$$
\begin{aligned}
L_{t, l} & =-\beta^{4} \sum_{P}\left(P^{2} \Delta_{t, l}-1\right) \\
& =-\beta^{4} \sum_{P} \int d x x \rho_{t, l}^{(\lambda)}(x, p)\left(\frac{P_{0}^{2}-p^{2}}{P_{0}^{2}-x^{2}}-1\right) .
\end{aligned}
$$

Next, with Eq. (B2), defining $\bar{\rho} \equiv\left(x^{2}-p^{2}\right) \rho$ [cf. Eq. (C1)] and using Eq. (A4), we may write

$$
L_{t, l}=\beta^{4} \frac{1}{2 \pi^{2}} \int_{0}^{\infty} d p p^{2} \int d x \bar{\rho}_{t, l}\left(x, p ; \lambda^{2} m^{2}\right)\left[\frac{1}{2}+n(x)\right] .
$$

Note that both, $\bar{\rho}$ and the square bracket, are odd functions of $x$. Let us split $\bar{\rho}$ into its leading part as given by Eqs. (C2) and (C3), and the rest $\bar{\rho}-\bar{\rho}^{\text {lead, }}$, which we call the soft part. Correspondingly, $L$ is written as $L^{\text {lead }}+L^{\text {soft }}$. Introducing an UV cutoff $\Lambda$, the leading parts may be written as

$$
\left.\begin{array}{c}
L_{t}^{\text {lead }} \\
L_{l}^{\text {lead }}
\end{array}\right\}=\left\{\begin{array}{c}
\lambda_{t}^{2} \\
2 \lambda_{l}^{2}
\end{array}\right\} \frac{\beta^{2} g^{2}}{12 \pi^{2}} \int_{0}^{\Lambda} d x x\left[\frac{1}{2}+n(x)\right]\left\{\begin{array}{c}
1-I(x) \\
I(x)
\end{array}, \quad I(x)=\int_{x}^{\Lambda} d p \frac{1}{p}\right.
$$

Equation (B5) is the right place deleting the " $\frac{1}{2}$ term" as discussed below Eq. (34) in the main text. But even under the control of the Bose function there remains a logarithmic divergent factor: namely,

$$
\kappa=\frac{\beta^{2}}{4 \pi^{2}} \int_{0}^{\Lambda} d x x n(x) \int_{0}^{\Lambda} d p \frac{1}{p} .
$$

Using Eq. (B6) we have $L_{t}^{\text {lead }}=\left(\frac{1}{24}-\kappa\right) \frac{1}{3} g^{2} \lambda_{t}^{2}$ and $L_{l}^{\text {lead }}$ $=\frac{2}{3} \kappa g^{2} \lambda_{l}^{2}$ which are the $\lambda^{2}$ terms in Eq. (60).

We turn to the soft parts of $L_{t, l}$, whose series might start with $g^{2} \lambda^{3}$. To prepare this $\lambda^{3}$ term, one may simply write
$T / x$ in place of the square bracket in Eq. (B4) (and, of course, the mentioned difference in place of $\bar{\rho}$ ). Using the sum rule (C5), one obtains

$$
\begin{aligned}
L_{l}^{\text {soft }} & =\frac{\beta^{3}}{2 \pi^{2}} \int_{0}^{\infty} d p p^{2} \int d x \frac{1}{x}\left[\bar{\rho}_{l}(x, p)-\bar{\rho}_{l}^{\text {lead }}(x, p)\right]_{m^{2} \rightarrow \lambda^{2} m^{2}} \\
& =-\frac{1}{4 \pi}\left(\frac{g \lambda_{l}}{\sqrt{3}}\right)^{3}
\end{aligned}
$$

But, through the above line and with a view to Eq. (C4), the transversal function $L_{t}^{\text {soft }}$ vanishes. This completes the derivation of Eq. (60). 
For the gutter problem of Sec. IV D we must still learn about the first nonvanishing piece of $L_{t}^{\text {soft }}$. Let us work with $\lambda_{t}=1$ and remember $m \rightarrow \lambda_{t} m$ at the end. We start from the full expression, but separate the cut and pole parts of the spectral densities. In particular, $\bar{\rho}_{t}^{\text {cut, lead }}$ means the second term in Eq. (C2), and $\bar{r}_{t}^{\text {lead }}=3 m^{2} / 4 p$ the prefactor of the $\delta$ functions. There is an exact expression (without index lead) to both. Then, three differences may be formed:

$$
\begin{aligned}
L_{t}^{\mathrm{soft}}\left(\lambda_{t}=\right. & 1)=\frac{\beta^{4}}{2 \pi^{2}} \int_{0}^{\infty} d p p^{2} \int d x\left[\frac{1}{2}+n(x)\right]\left\{2\left(\overline{r_{t}}-\bar{r}_{t}^{\text {lead }}\right)\right. \\
& \times \delta\left(x-\omega_{t}\right)+\bar{\rho}_{t}^{\text {cut }}-\bar{\rho}_{t}^{\text {cut, lead }} \\
& \left.+2 \bar{r}_{t}^{\text {lead }}\left[\delta\left(x-\omega_{t}\right)-\delta(x-p)\right]\right\}
\end{aligned}
$$

where $\omega_{t}=\omega_{t}(p)$ is the transversal plasma frequency, to be obtained by solving $\omega_{t}^{2}=p^{2}+\Pi_{t}\left(\omega_{t}, p\right)$. We now notice that $x, p$ are restricted to soft values by the above first two differences, but not by the third one. So, in front of the first two, we may still use the $T / x$ approximation. Note that $\frac{1}{2}+n(x)-T / x=\beta x / 12+O\left(\beta^{2} x^{2}\right)$. Hence, for $\beta x \sim g$ this difference is by two $g$ powers smaller than $T / x \sim 1 / g$. It might contribute to $L$ only at $g^{5}$. Working this way, the sum rule helps again to get rid of $\overline{\rho_{t}}$ and $\overline{r_{t}}$ :

$$
L_{t}^{\mathrm{soft}}\left(\lambda_{t}=1\right)=\frac{\beta^{3} 3 m^{2}}{4 \pi^{2}} \int_{0}^{\infty} d p\left(1-\frac{p}{\omega_{t}}+\beta p\left[n\left(\omega_{t}\right)-n(p)\right]\right) .
$$

For convenience, this can be further rewritten by introducing $\omega=\omega_{t}$ as the integration variable [and by once more replacing $n(p) \rightarrow T / p$ in a soft term-this time required for consistency]:

$$
L_{t}^{\mathrm{soft}}\left(\lambda_{t}=1\right)=\frac{\beta^{3} 3 m^{2}}{4 \pi^{2}} \int_{m}^{\infty} d \omega[1-\beta \omega n(\omega)]\left(1-\frac{p(\omega)}{\omega \omega^{\prime}}\right)
$$

with $\omega^{\prime}$ the derivative of $\omega_{t}$ with respect to $p$, and $p$ being $p(\omega)$. The square bracket starts as $\frac{1}{2} \beta \omega$ for small $\beta \omega$, its saturation at 1 being never reached because the round bracket sets the limit. It starts with $1 / 6$ (at $\omega \rightarrow m$ ) and goes as $(9 / 4) m^{4} \omega^{-4} \ln (\omega / m)$ for large $\omega$ (with such details taken from Appendix B of [23]). Hence Eq. (B10) is indeed of order $g^{4}$ in magnitude. Going to $\lambda_{t} \neq 1$ simply amounts to $m \rightarrow \lambda_{t} m$ in Eq. (B10). But note that this scaling also changes the definition of, e.g., $\omega_{t}$, which now is the transversal plasma frequency as if $m$ were $\lambda_{t} m$.

What we really need in the main text, is not $L_{t}$ itself but the first factor in Eq. (65). The operation there, fortunately, eliminates the above last integration:

$$
\frac{2}{\lambda_{t}} L_{t}-\partial_{\lambda_{t}} L_{t}=\frac{g^{3} \lambda_{t}^{2}}{216 \pi^{2}}\left[1-\frac{g \lambda_{t}}{3} n\left(\frac{T g \lambda_{t}}{3}\right)\right]
$$

This "first factor"' is thus positive, and it behaves as $\sim g^{4} \lambda_{t}^{3}$ for small $g$. Just these properties were used in the main text below Eq. (65) to reach the long bathtub.

\section{APPENDIX C}

Here we collect a few special details on the spectral densities $\rho_{t}$ and $\rho_{l}$ which were needed in Appendix B. There we had to learn on the product

$$
\bar{\rho}(x, p) \equiv\left(x^{2}-p^{2}\right) \rho(x, p)
$$

and its asymptotic forms at large $p$ argument $\left(p^{2} \gg m^{2}\right)$ :

$$
\begin{gathered}
\bar{\rho}_{t}^{\text {lead }}=\frac{3 m^{2}}{4 p}[\delta(x-p)-\delta(x+p)]-\frac{3 m^{2}}{4 p^{3}} x \theta\left(p^{2}-x^{2}\right) \\
\bar{\rho}_{l}^{\text {lead }}=+\frac{3 m^{2}}{2 p^{3}} x \theta\left(p^{2}-x^{2}\right) .
\end{gathered}
$$

These leading terms are readily obtained from the full expressions as given in Appendix B of [23]. One may check Eqs. (C2) and (C3) by using it in the $\bar{\rho}$ sum rules and thereby producing, in each case, the term of highest $p$ power to the right. The exact $\bar{\rho}$ sum rules read

$$
\begin{gathered}
\int d x\left\{\begin{array}{c}
1 / x \\
x \\
x^{3} \\
x^{5} \\
x^{7}
\end{array}\right\} \bar{\rho}_{t}(x, p)=\left\{\begin{array}{c}
0 \\
m^{2} \\
\frac{6}{5} p^{2} m^{2}+m^{4} \\
\frac{9}{7} p^{4} m^{2}+\frac{12}{5} p^{2} m^{4}+m^{6} \\
\frac{4}{3} p^{6} m^{2}+\frac{702}{175} p^{4} m^{4}+\frac{18}{5} p^{2} m^{6}+m^{8}, \\
3 m^{2} /\left(3 m^{2}+p^{2}\right) \\
m^{2} \\
\frac{3}{5} p^{2} m^{2}+m^{4} \\
\frac{3}{7} p^{4} m^{2}+\frac{6}{5} p^{2} m^{4}+m^{6} \\
x^{3} \\
x^{5} \\
x^{7}
\end{array}\right\} \bar{\rho}_{l}(x, p)=\left\{\begin{array}{c}
1 / x \\
\frac{1}{3} p^{6} m^{2}+\frac{213}{175} p^{4} m^{4}+\frac{9}{5} p^{2} m^{6}+m^{8} .
\end{array}\right.
\end{gathered}
$$

They derive through Eq. (C1) from the sum rules of ordinary densities: 


$$
\begin{gathered}
\int d x\left\{\begin{array}{c}
1 / x \\
x \\
x^{3} \\
x^{5} \\
x^{7} \\
x^{9}
\end{array}\right\} \rho_{t}(x, p)=\left\{\begin{array}{c}
1 / p^{2} \\
1 \\
p^{2}+m^{2}
\end{array}\right. \\
\int d x\left\{\begin{array}{c}
p^{4}+\frac{11}{5} p^{2} m^{2}+m^{4} \\
1 / x \\
x \\
x^{3} \\
x^{5} \\
x^{7} \\
x^{9}
\end{array}\right\} \rho_{l}(x, p)=\left\{\begin{array}{c}
p^{6}+\frac{122}{35} p^{4} m^{2}+\frac{17}{5} p^{2} m^{4}+m^{6} \\
p^{8}+\frac{506}{105} p^{6} m^{2}+\frac{1297}{175} p^{4} m^{4}+\frac{23}{5} p^{2} m^{6}+m^{8}, \\
1 /\left(3 m^{2}+p^{2}\right) \\
1 \\
p^{2}+m^{2} \\
p^{4}+\frac{8}{5} p^{2} m^{2}+m^{4} \\
p^{6}+\frac{71}{35} p^{4} m^{2}+\frac{11}{5} p^{2} m^{4}+m^{6} \\
p^{8}+\frac{248}{105} p^{6} m^{2}+\frac{598}{175} p^{4} m^{4}+\frac{14}{5} p^{2} m^{6}+m^{8},
\end{array}\right.
\end{gathered}
$$

and these, in turn, are derived along the lines given in [20].

[1] R. P. Feynman, Nucl. Phys. B188, 479 (1981); Variational Calculations in Quantum Field Theory, edited by L. Polley and D. E. L. Pottinger (World Scientific, Singapore, 1988).

[2] K. Huang, Statistical Mechanics (Wiley, New York, 1987).

[3] W. Brenig, Statistische Theorie der Wärme (Springer, Berlin, 1975).

[4] S. V. Tjablikov, Methods in Quantum Theory of Magnetism (Plenum, New York, 1967).

[5] P. M. Chaikin and T. C. Lubensky, Principles of Condensed Matter Physics (Cambridge University Press, Cambridge, England, 1995).

[6] R. Manka, Ann. Phys. (N.Y.) 171, 1 (1986).

[7] G. A. Hajj and P. M. Stevenson, Phys. Rev. D 37, 413 (1988).

[8] B. Alles, R. Munoz-Tapia, and R. Tarrach, Ann. Phys. (N.Y.) 204, 432 (1990).

[9] T. Hatsuda and T. Kunihiro, Phys. Rep. 247, 221 (1994).

[10] R. P. Feynman, Statistical Mechanics (W. A. Benjamin, Massachusetts, 1972).

[11] R. P. Feynman, Phys. Rev. 97, 660 (1954).

[12] H. Kleinert, Path Integrals in Quantum Mechanics, Statistics and Polymer Physics (World Scientific, Singapore, 1990).

[13] U. Kerres, G. Mack, and G. Palma, Nucl. Phys. B467, 510 (1996).

[14] W. Rühl, Z. Phys. C 32, 265 (1986).

[15] Y. Schröder, diploma thesis (in German), Hannover University, 1995).

[16] E. Braaten and R. D. Pisarski, Nucl. Phys. B337, 569 (1990); B339, 310 (1990); J. Frenkel and J. C. Taylor, ibid. B334, 199 (1990).

[17] J. C. Taylor and S. M. Wong, Nucl. Phys. B346, 115 (1990); J. Frenkel and J. C. Taylor, ibid. B374, 156 (1992); R. D. Pisarski, in From Fundamental Fields to Nuclear Phenomena, edited by J. A. McNeil and C. E. Price (World Scientific, Singapore, 1991); E. Braaten and R. D. Pisarski, Phys. Rev. D 45, R1827 (1992); R. Efraty and V. P. Nair, ibid. 47, 5601 (1993).
[18] R. Ibanez-Meier, I. Stancu, and P. M. Stevenson, Z. Phys. C 70, 307 (1996).

[19] U. Kraemmer, A. K. Rebhan, and H. Schulz, Ann. Phys. (N.Y.) 238, 286 (1995); in From Thermal Field Theory to Neural Networks, edited by P. Aurenche, P. Sorba, and G. Veneziano (World Scientific, Singapore, 1996).

[20] H. Schulz, Phys. Lett. B 291, 448 (1992).

[21] J. I. Kapusta, Finite-Temperature Field Theory (Cambridge University Press, Cambridge, England, 1989).

[22] P. Landsman and Ch. van Weert, Phys. Rep. 145, 141 (1987).

[23] H. Schulz, Nucl. Phys. B413, 353 (1994).

[24] M. Kreuzer, A. Rebhan, and H. Schulz, Phys. Lett. B 244, 58 (1990).

[25] C. Itzykson and J.-B. Zuber, Quantum Field Theory (McGrawHill, New York, 1985).

[26] C. W. Bernard, Phys. Rev. D 9, 3312 (1974).

[27] Ch. Blatter, Analysis 1 (Springer, Berlin, 1991).

[28] R. E. Peierls, Phys. Rev. 54, 918 (1938).

[29] R. Ibanez-Meier, L. Polley, and U. Ritschel, Phys. Lett. B 279, 106 (1992).

[30] L. Dolan and R. Jackiw, Phys. Rev. D 9, 3320 (1974).

[31] P. Arnold and O. Espinosa, Phys. Rev. D 47, 3546 (1993); P. Arnold and C. Zhai, ibid. 50, 7603 (1994); C. Zhai and B. Kastening, ibid. 52, 7232 (1995).

[32] K. Kajantie et al., Nucl. Phys. B458, 90 (1996).

[33] E. Braaten, Phys. Rev. Lett. 74, 2164 (1995); E. Braaten and A. Nieto, Phys. Rev. D 51, 6990 (1995); Phys. Rev. Lett. 74, 3530 (1995).

[34] P. Arnold and L. G. Yaffe, Phys. Rev. D 52, 7208 (1995).

[35] W. Buchmüller et al., Ann. Phys. (N.Y.) 234, 260 (1994); W. Buchmüller and O. Philipsen, Nucl. Phys. B443, 47 (1995); Phys. Lett. B 354, 403 (1995); W. Buchmüller, Z. Fodor, and A. Hebecker, Nucl. Phys. B447, 317 (1995).

[36] F. Flechsig, A. Rebhan, and H. Schulz, Phys. Rev. D 52, 2994 (1995).

[37] O. K. Kalashnikov and V. V. Klimov, Phys. Lett. 95B, 423 (1980). 
[38] S. K. Blau, M. Visser, and A. Wipf, Int. J. Mod. Phys. A 4, 1467 (1989); 6, 5409 (1991); I. Sachs and A. Wipf, Jena Report No. TPI 95/2 972 (unpublished).

[39] W. Dittrich and M. Reuter, Selected Topics in Gauge Theories, Lecture Notes in Physics No. 244 (Springer, Berlin, 1986).

[40] F. Flechsig and H. Schulz, Phys. Lett. B 349, 504 (1995).

[41] V. P. Nair, Phys. Lett. B 352, 117 (1995); G. Alexanian and V. P. Nair, ibid. 352, 435 (1995); R. Jackiw and S. Pi, ibid. 368, 131 (1996); J. M. Cornwall and W.-S. Hou, Phys. Rev. D 34,
585 (1986).

[42] U. M. Heller, F. Karsch, and J. Rank, Phys. Lett. B 355, 511 (1995).

[43] J-P Blaizot and E. Iancu, Nucl. Phys. B459, 559 (1996); Phys. Rev. Lett. 76, 3080 (1996).

[44] I. Stancu and P. M. Stevenson, Phys. Rev. D 42, 2710 (1990); R. Ibanez-Meier, Ph.D. thesis, Rice University, 1992.

[45] E. R. Hansen, A Table of Series and Products (Prentice-Hall, Englewood Cliffs, NJ, 1975). 